\title{
Recent Progress in Hydrogen-Bonded $\pi$-Conjugated Systems Displaying J-Type Aggregates
}

\author{
Nelson Ricardo Ávila-Rovelo ${ }^{a}$ (D) \\ Amparo Ruiz-Carretero*a \\ a Institute Charles Sadron, CNRS, University of Strasbourg, 23 Rue du Loess, BP 84047, \\ 67034 Strasbourg, Cedex 2, France \\ amparo.ruiz@ics-cnrs.unistra.fr
}

Received: 26.12.2019

Accepted after revision: 10.02.2020

DOI: 10.1055/s-0040-1708502; Art ID: 190027rev

License terms: (c)

(c) 2019. The Author(s). This is an open access article published by Thieme under the terms of the Creative Commons Attribution-NonDerivative-NonCommercial-License, permitting copying and reproduction so long as the original work is given appropriate credit. Contents may not be used for commercial purposes, or adapted, remixed, transformed or built upon. (https://creativecommons.org/licenses/by-nc-nd/4.0/)

Abstract Supramolecular approaches are of great interest in the design of functional materials. The types of aggregates arising from different noncovalent interactions endow materials with intriguing properties. In this sense, J-type aggregates are very attractive due to their unique optical properties and capacity to transport excitons. These features make them great candidates in the design of materials for organic electronic devices. Furthermore, the incorporation of additional hydrogen-bonding functionalities provides J-aggregates with superior directionality and connection among the different $\pi$-conjugated cores. The control over the formation of $\mathrm{H}$-bonds to achieve functional aggregates is therefore a promising strategy towards controlled structures with specific functions.

This review outlines the most relevant and recent works of $\pi$-conjugated systems exhibiting J-type aggregates resulting from hydrogen-bonding interactions. Different types of hydrogen-bonding functionalities will be discussed together with their roles in the aggregate properties, their impact in the optoelectronic properties, the self-assembly mechanisms, and their applications in organic electronics.

Key words Hydrogen-bonding, J-aggregates, organic electronics

\section{Introduction}

Intermolecular interactions guide the formation of supramolecular structures with intriguing properties and functions. ${ }^{1-5}$ They can play an important role in the design of supramolecular materials with electronic applications. ${ }^{6}$ Among the many weak intermolecular interactions found in

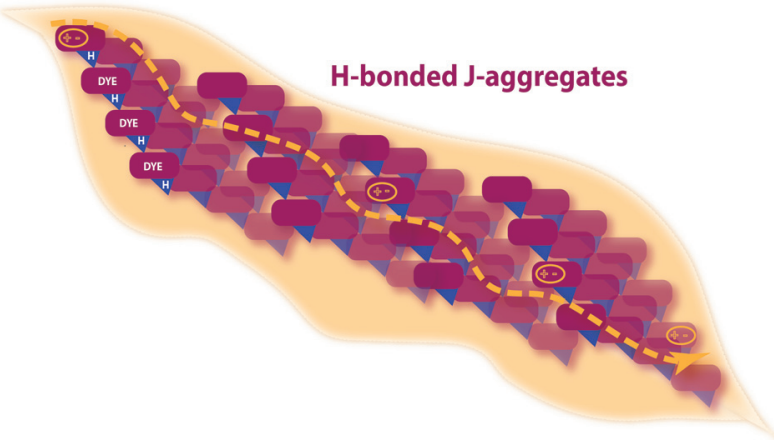

organic materials, hydrogen bonds (H-bonds) provide directionality and selectivity to supramolecular assemblies. $^{7-11}$ Regarding organic semiconductors, $\pi-\pi$ stacking interactions are predominant due to the presence of aromatic moieties within their molecular structures. Nevertheless, when H-bonds are also incorporated in the semiconductors, different aggregates can arise from the interplay between $\pi-\pi$ stacking and H-bonding, making great impact on the optoelectronic properties. ${ }^{11-14}$ Particularly, J-type aggregates, named after one of their first discoverers (Edwin E. Jelley), ${ }^{15}$ are very interesting due to their exciting optical properties, very different from the properties of the monomers in the nonaggregated state.

J-type aggregates were first reported by Günter Scheibe ${ }^{16}$ and Edwin E. Jelley ${ }^{15}$ in the 1930s as a particular aggregation form of chromophores, where the appearance of an additional narrow absorption band, bathochromically shifted with respect to the monomer absorption band with a narrow fluorescence band and a small Stokes shift, was observed (Figure 1a). This behavior was initially found in aqueous solutions of 1,1'-diethyl-2,2'-cyanine chloride abbreviated as PIC (Figure 1a). Scheibe interpreted this result as the polymerization of the dye, meaning supramolecular polymerization due to noncovalent interactions.

Even though different models for the arrangement of dyes within a J-aggregate have been proposed, it is generally accepted that the dyes are usually positioned in a "brickwork" fashion where dyes are significantly slipped with respect to each other, rather than in a face-to-face disposition (Figure 1b). ${ }^{17}$ Therefore, the coupling of the transition dipole moments is very effective, allowing the communication among a large amount of dye molecules and resulting in exciton delocalization and energy migration up to micron distances. ${ }^{18}$

On the other hand, aggregates showing hypsochromically shifted absorption bands, with respect to the monomer absorption, are called H-aggregates (from hypsochromic) 
Biographical Sketch
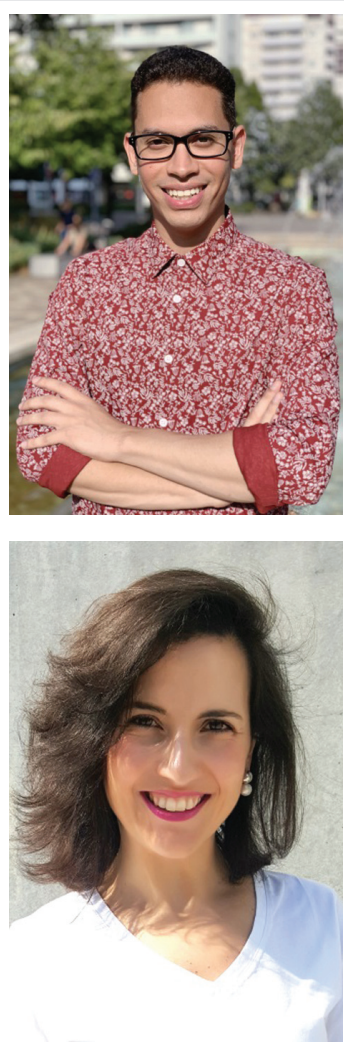

Nelson Ricardo Ávila-Rovelo was born in Tegucigalpa, Honduras in 1992. He received his bachelor's degree in chemical engineering at the Universidad Nacional Autónoma de Honduras. He then obtained two master degrees, one in polymer science and the other in sustainable materials, from the consortium held between Université de Strasbourg and Albert-Ludwigs-Universität Freiburg. He is currently a first-year PhD student at Institute Charles Sadron (CNRS) in Strasbourg (France), un- der the supervision of A. RuizCarretero, where he is performing his research on the in situ control over morphology in organic electronic devices containing $\mathrm{H}$-bonds.
Amparo Ruiz-Carretero is a "Chargé de Recherche" at Institute Charles Sadron (CNRS) in Strasbourg (France). She studied Chemistry at the University of Castilla-La Mancha (Spain), where she obtained her PhD in 2009 under the supervision of
Prof. A. de la Hoz and Dr. A. Sánchez. She spent a big part of her PhD research in the group of Prof. E. W. Meijer and Prof. A. Schenning. After postdocs with the groups of Prof. S. Stupp (Northwestern University) and Prof. L. De Cola (University of
Strasbourg), she obtained her current CNRS position in 2015. Her research interests include the study of hydrogen-bonded semiconductors, supramolecular chirality, and the influence of magnetic fields in organic electronics. and usually characterized by having low or no fluorescence. ${ }^{19}$ In this case, the molecules are stacked in a face-toface manner, resulting in emission quenching (Figure 1c). In some cases, such aggregates can also be stabilized by introducing $\mathrm{H}$-bonding groups. ${ }^{20}$

The interesting optical properties of the PIC dye in its aggregated state were revealed when it was applied as a spectral sensitizer in photography. ${ }^{21}$ Sensitizing dyes are physically adsorbed on silver halides, forming J-aggregates

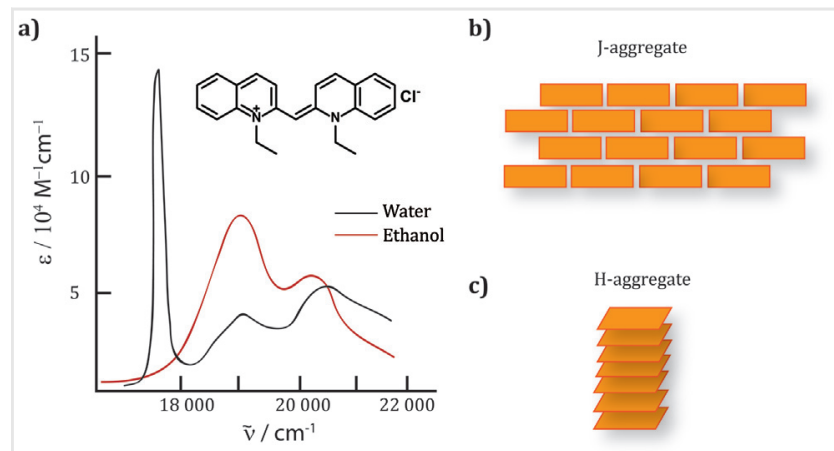

Figure 1 (a) PIC chloride molecular structure and the spectroscopic changes from ethanol to water solution, showing the typical J-aggregate red-shifted band. (b) Brick-work disposition of dyes within J-aggregates. (c) Face-to-face stack of molecules in $\mathrm{H}$-aggregates. that make possible the migration of an excited dye molecule until it finds a suitable place for charge separation and electron transfer.

After the discovery of J-type aggregates in PIC chloride, many other dyes exhibiting the same kind of aggregates have been studied, including merocyanines, ${ }^{22}$ squaraines, ${ }^{23}$ perylene bisimides (PBIs), ${ }^{19,24,25}$ porphyrins, ${ }^{26}$ tetrathiofulvalenes (TTFs), ${ }^{27}$ and diketopyrrolopyrroles (DPPs), ${ }^{28,29}$ among others. J-type aggregates can be found in assemblies of dyes formed via different noncovalent interactions; however, in this review the most recent and relevant cases of $\mathrm{H}$-bonded synthetic $\pi$-conjugated systems displaying $\mathrm{J}$ type aggregates will be discussed. Nevertheless, not only Jtype aggregates have been studied in synthetic dyes, but they are also found in natural light-harvesting (LH) complexes. This is the case, for example, of chlorosome LH complexes found in photosynthetic bacteria, where arrays of bacteriochlorophyll (BChl) derivatives are arranged via $\mathrm{H}$-bonding, metal-ligand coordination, and $\pi-\pi$ stacking to form dye assemblies displaying J-type excitonic coupling. ${ }^{30,31}$ Inspiration from these systems has been crucial to understand the properties of J-type aggregates and extend the studies to synthetic dyes. One of the most studied biomimicking LH complexes uses meso-tetrakis(4sulfonatophenyl)porphyrin (TPPS), ${ }^{32}$ which upon changing 
the $\mathrm{pH}$ in solution has the ability to self-assemble into J- and $\mathrm{H}$-type aggregates. At low $\mathrm{pH}(<2)$, TPPS forms J-aggregates forming nanorods that are $3-5 \mathrm{~nm}$ in height and reach $50-2,000 \mathrm{~nm}$ in length, where charges can be efficiently transported. These results highlight the unique optical properties and capacity to transport excitons of J-aggregates. Since it was discovered that LH complexes in natural photosynthetic organisms and plants are dictated by processes similar to those happening in synthetic dyes, the interest in the properties of self-assembled $\pi$-conjugated systems has increased. Excited states in J-type aggregates are Frenkel excitons (excitations where holes and electrons are tightly bound $)^{33,34}$ delocalized over many dye molecules (up to 50-100) depending on the conditions (morphology, disorder, and temperature). ${ }^{35,36}$ Such delocalization facilitates energy migration within the aggregate, making $\pi$-conjugated systems that form J-aggregates great candidates to be used in energy-related materials. Many experimental and theoretical studies have been carried out on the dimensionality and morphology of J-aggregates in order to know the coherence length and the number of chromophores among which the excitons can be delocalized, ${ }^{17,35,37,38}$ finding that it is possible to reach up to 70-100 nm. ${ }^{39}$

Such studies show that highly organized self-assembled systems can be the next generation of organic materials for solar-cell applications and organic electronics in general. Even though initial studies were done in polar solvents with high salt concentrations to promote the formation of aggregates, many examples of aggregates formed in apolar solvents have been described, making them the most interesting cases for organic electronics purposes. The incorporation of additional H-bonding functionalities provides the aggregates with superior directionality and connection among the different $\pi$-cores. The control over the formation of $\mathrm{H}$-bonds to achieve functional aggregates is therefore a promising strategy towards controlled structures with specific functions.

This review is not meant to be an exhaustive work on Jtype aggregates but more to collect the most relevant and recent progresses on systems where such types of aggregates arise from $\mathrm{H}$-bonding interactions. The readers are referred to broader and more exhaustive reviews on Jtype aggregates, ${ }^{19,40,41}$ since the focus of this review is on $\mathrm{H}$ bonded systems and electronic applications. By citing these reviews we would like to raise awareness regarding the interpretation of J-aggregates. Even though we have followed the respective authors' interpretations considering in some cases only a red-shift in the absorption spectrum as the single criterion to identify a J-aggregate, other criteria such as the narrowing of the absorption and fluorescence bands, a decrease in Stokes shift compared to the monomeric species, and a decrease in fluorescence lifetime should also be taken into account. Furthermore, when the strength of the intermolecular (excitonic) coupling is similar to the electronic-vibrational (vibronic) coupling, the results from Kasha's theory (H-aggregates show blue-shifts and Jaggregates red-shifts) can be modified. According to Spano, ${ }^{19}$ under these conditions, $\mathrm{H}$ - and J-type aggregates can be differentiated by calculating the ratio of the first two vibronic peaks of the absorption spectrum, which increases with increasing excitonic coupling (J-aggregate) and vice versa. Más-Montoya et $a l^{42}$ showed this effect in small thiophene-pyridine DPP derivatives, where they followed the evolution of the $0-0 / 0-1$ vibronic peak ratio in the absorption spectra. In addition, they observed that the derivatives that self-assembled into J-type aggregates have enhanced photovoltaic performance. Very recent works by Zhong et al. ${ }^{43}$ also illustrated the divergences from the Kasha model in highly polarizable molecules.

Even though the recent progress in H-bonded systems displaying J-aggregates is the aim of this review, we consider that earlier works are needed to be highlighted in order to put this review into context. The interesting optoelectronic properties and the applications in organic electronics will be reviewed.

\section{Amide-Containing Systems}

Amide functional groups are probably the best known $\mathrm{H}$ bond forming groups. ${ }^{44}$ The well-known synthetic procedures to introduce them into any type of molecule and their easy characterization by spectroscopy techniques, such as infrared, make them the perfect candidates to study selfassembly processes. Conjugated systems containing one or two amide bonds will be discussed in this part of the review. Tsai et al. ${ }^{45}$ have shown a hairpin-shaped molecule containing a sexithiophene unit as the electroactive segment attached to trans-1,2-diamidocyclohexane (DACH) as the self-assembly motif (Figure 2a). In this case, the coexistence of $\mathrm{H}$ - and J-aggregates results in the broadening of the wavelength spectral range of optical absorption, and in the formation of semiconducting nanowires (Figure 2a). The authors compared the UV/Vis spectra of the hairpin-shaped molecule in toluene straight after solution (fresh) preparation and after 48 hours of aging, finding striking differences in the absorption properties. In addition to the original absorption band at $\lambda=423 \mathrm{~nm}$ (Figure 2b), a red-shifted band at $500 \mathrm{~nm}$ appeared, while another band previously present at $313 \mathrm{~nm}$ became more predominant (Figure 2b,c). Organic field-effect transistors (OFETs) were fabricated with the hairpin-shaped molecule to evaluate the charge mobility. The hole mobility $\left(\mu_{\mathrm{h}}\right)$ values found in devices fabricated from toluene solutions (self-assembly promoting solvent) were roughly 1 order of magnitude higher $\left(\mu=3.46 \times 10^{-6} \mathrm{~cm}^{2} \mathrm{~V}^{-1} \mathrm{~s}^{-1}\right)$ than the values found for the devices made from nonassembling 
a)

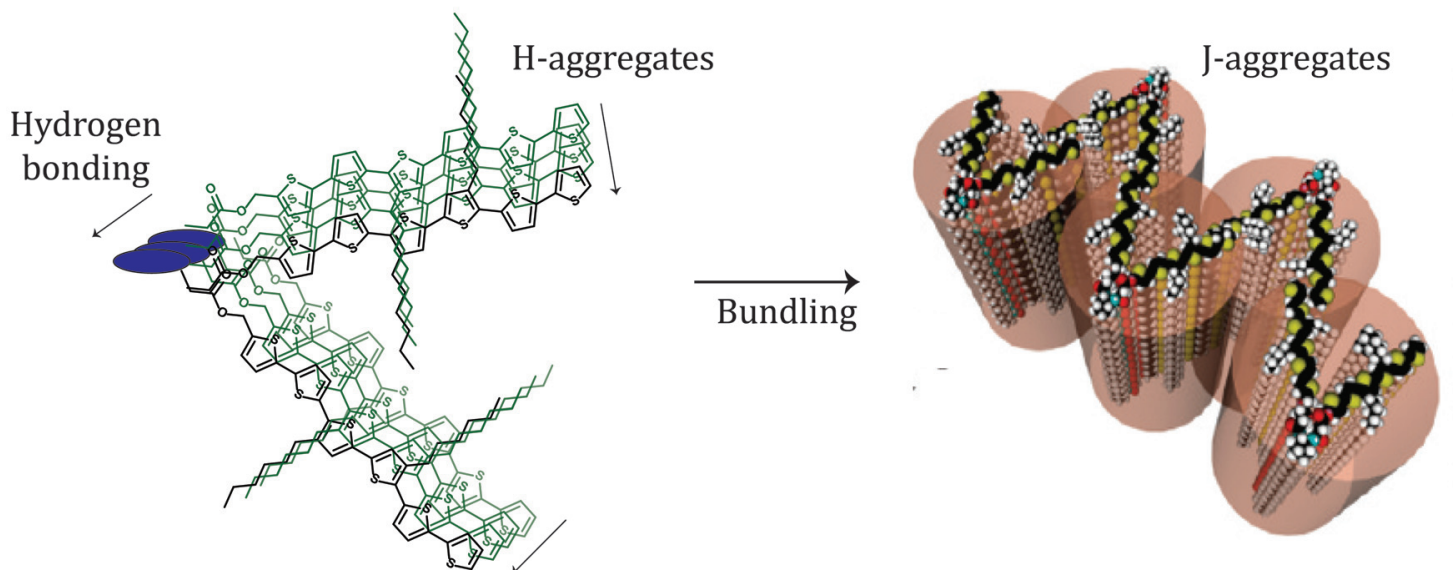

b)

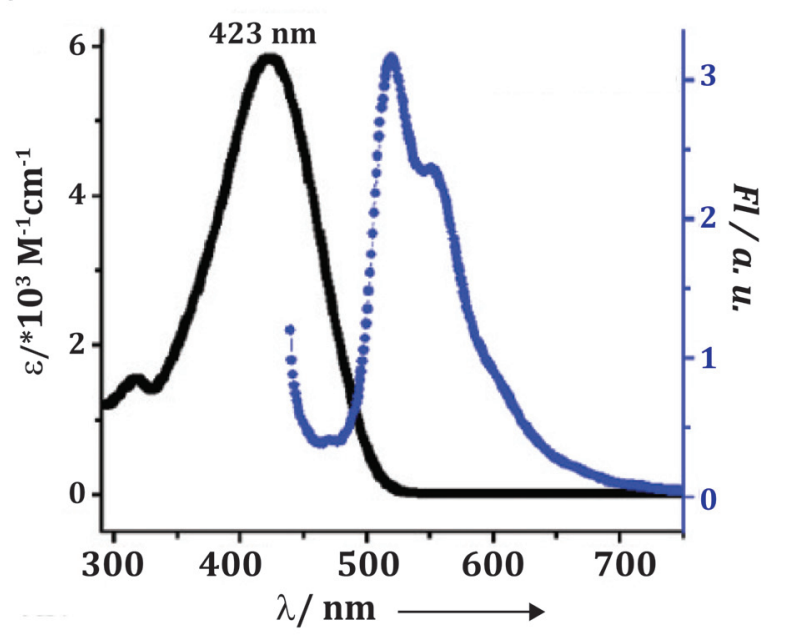

c)

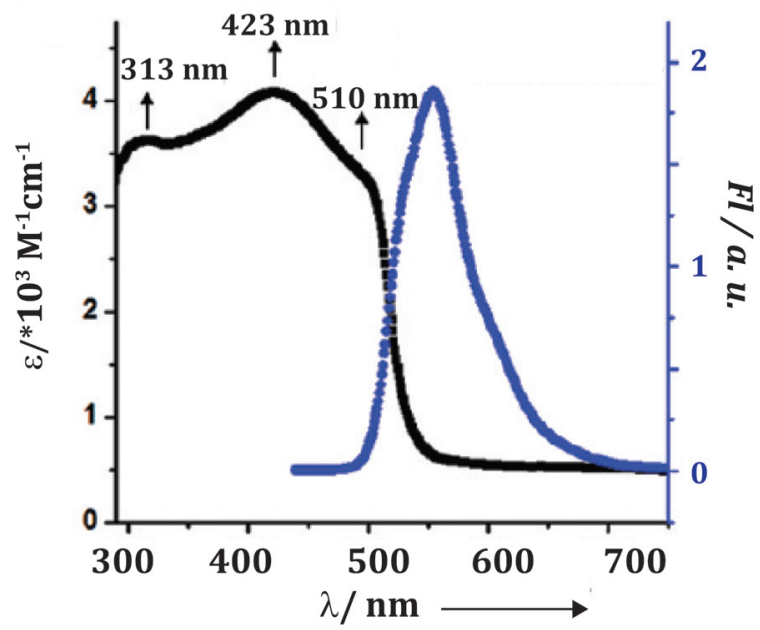

Figure 2 (a) Schematic representation of the self-assembly process of the hairpin-shaped sexithiophene derivative forming $\mathrm{H}$-and J-type aggregates. (b) Absorption and emission spectra of the hairpin molecule freshly prepared. (c) Absorption and emission spectra of the hairpin molecule after 48 hours of aging in toluene. Adapted with permission from Ref. 45. Copyright 2010 American Chemical Society.

solvents, such as chlorobenzene and $\boldsymbol{o}$-chlorobenzene $\left(\mu=9.42 \times 10^{-7}\right.$ and $1.79 \times 10^{-7} \mathrm{~cm}^{2} \mathrm{~V}^{-1} \mathrm{~s}^{-1}$, respectively). These results could be explained by the broadening of the absorption due to the $\mathrm{H}$ - and J-type aggregates that facilitate charge transport along the nanowires and between nanowires. In a following work, the same group reported the applications of the hairpin-shaped derivative in organic photovoltaic devices. ${ }^{46}$ In this case, the hairpin-shaped sexithiophene derivative was used as a donor material in bulk-heterojunction solar cells in combination with phenyl$\mathrm{C}_{61}$-butyric acid methyl ester $\left(\mathrm{PC}_{61} \mathrm{BM}\right)$ as the acceptor material. The grooved nature of the hairpin-shaped molecule allows the interaction with $\mathrm{PC}_{61} \mathrm{BM}$ derivatives in a receptor-ligand manner. The authors found that J-type aggregates led to the formation of bundles of hairpin nanofibers. The J-aggregate signals disappeared upon interaction with $\mathrm{PC}_{61} \mathrm{BM}$ molecules as a proof of the disentanglement of nanofibers upon interaction with the acceptor molecules. Similar results were observed by Ghosh et al. ${ }^{47}$ They found Davydov splitting ${ }^{34}$ in a DPP system symmetrically functionalized with amide bonds (Figure 3a). The authors observed the presence of $\mathrm{H}$ - and J-type aggregates upon self-assembly. ${ }^{47}$ Dilute solutions in chloroform and toluene showed absorption spectra of the monomeric species of DPP-Amide (Figure 3b, blue spectrum), exhibiting two main absorption bands, one at $428 \mathrm{~nm}$, corresponding to a $\pi-\pi^{*}$ transition, and a broad band with a maximum at $628 \mathrm{~nm}$, attributed to intermolecular charge transfer between the electron-rich (oligothiophene) part of the molecule and the electron-poor DPP. In contrast, under aggregation conditions, the absorption spectrum differs from the one at low concentration. In this 
a)

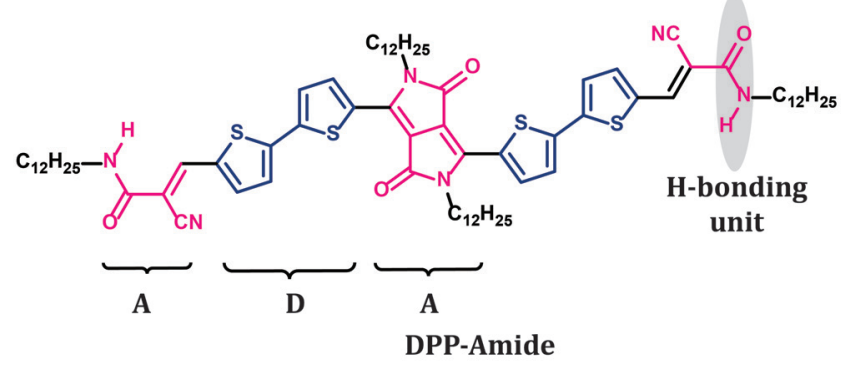

b)

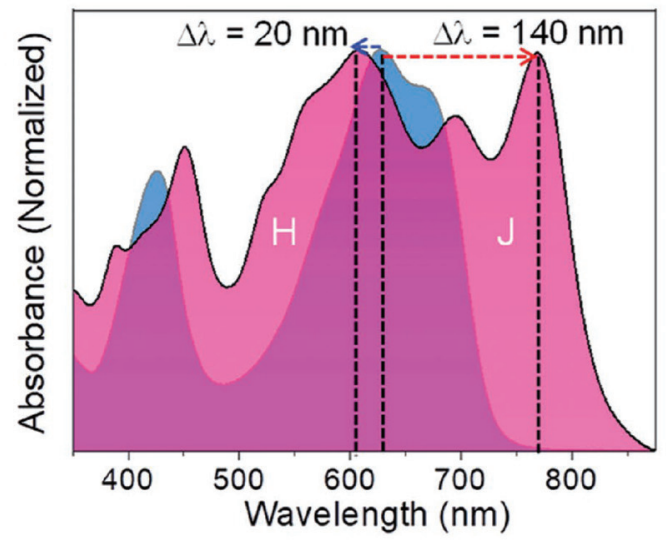

c)

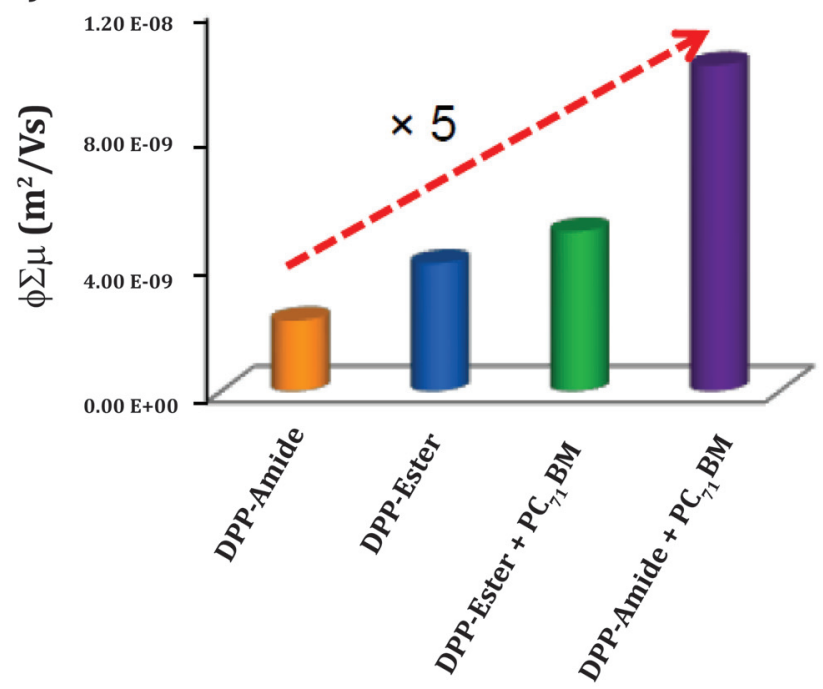

Figure 3 (a) Molecular structure of DPP-Amide. (b) Absorption spectra of DPP-Amide in its disassembled (blue) and assembled (pink) states. (c) Photoconductivity values of DPP-Amide, DPP-Ester, and their blends with PC $_{71}$ BM. Images (a) and (b) have been adapted with permission from Ref. 47. Copyright 2017 John Wiley and Sons. Image (c) has been adapted with permission from Ref. 48. Copyright 2018 John Wiley and Sons.

case, new bands appear at higher and lower energies, being $\mathrm{H}$ - and J-type aggregates, respectively, and achieving spectral coverage in the visible region and transparency in the near-infrared (NIR) region (Figure 3b, pink spectrum). As a proof of concept, the authors fabricated a self-standing PDMS (polydimethylsiloxane) NIR-transmitting optical filter by blending DPP-Amide self-assembled fibers at different concentrations. The authors successfully demonstrated the use of the filters in night vision, anticounterfeiting, and forensic applications.

Subsequently, DPP-Amide was studied in combination with $\mathrm{PC}_{71} \mathrm{BM}$ in hybrid organogels exhibiting high photoconductivity. ${ }^{48}$ DPP-Amide exhibits photoinduced electron transfer (PET) only in the assembled state in the presence of $\mathrm{PC}_{71} \mathrm{BM}$, leading to enhanced photoconductivity. DPPAmide showed photoconductivity only in the assembled state, finding a fivefold enhancement when blended with $\mathrm{PC}_{71} \mathrm{BM}$ (Figure $3 \mathrm{c}$ ). An analogue derivative containing ester groups was synthesized as a control molecule (DPP-Ester), which did not show J-aggregates and did not form organo- gels. The photoconductivity values for DPP-Ester were slightly higher than those for DPP-Amide, but when DPPEster was blended with $\mathrm{PC}_{71} \mathrm{BM}$, the photoconductivity value did not change. This result highlights the important role of having $\mathrm{H}$-bonds in the molecular structure, which results in J-aggregates leading to higher photoconductivity.

The field of perovskite photovoltaic devices has also benefitted from the presence of materials showing Jaggregates. For instance, Kaneko et al. ${ }^{49}$ have shown $\mathrm{H}$ bonded TTF derivatives containing amide groups that have been used as dopant-free hole transport materials in perovskite solar cells. In this case, the H-bonded TTF derivatives had electrical conductivity values higher than spiro-OMeTAD (2,2',7,7'-tetrakis[N,N-di(4-methoxyphenyl) amino]-9,9'-spirobifluorene, which is the most usual hole transport material. J-type aggregates were found in $\mathrm{H}$ bonded TTF thin films leading to the formation of nanofibers with enhanced mobility.

Many examples of PBI derivatives with pending amide groups exhibiting J-aggregates have been reported. 
Würthner's group has shown multiple examples of selfassembled PBIs ${ }^{50}$ containing amide bonds as well as other self-assembly motifs. Back in 2008 they showed a study on how to control $\mathrm{H}$ - and J-type homo- and heteroaggregates in amide-containing PBIs. For this purpose, they synthesized several PBI derivatives (Figure 4a) with the same core and amide groups as H-bonding motifs and changed the alkyl chains. ${ }^{51}$ The authors found that the steric effects in the

a)

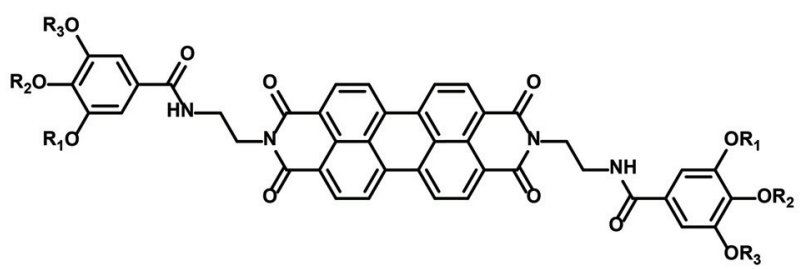

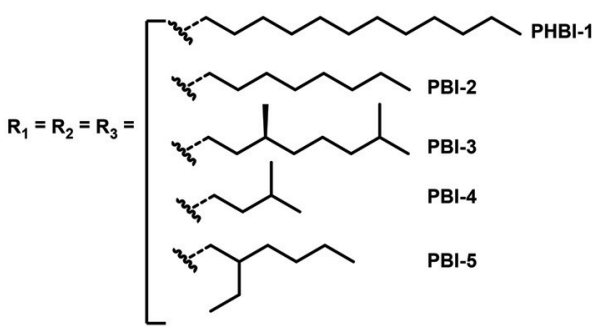

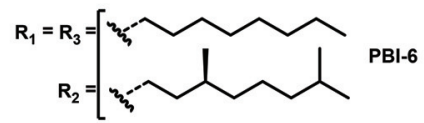

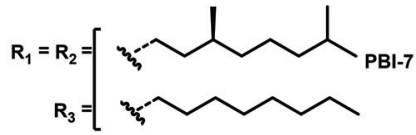

b)

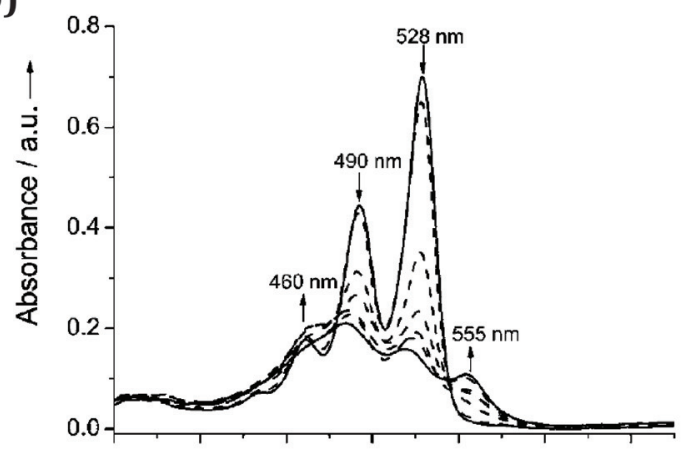

c)

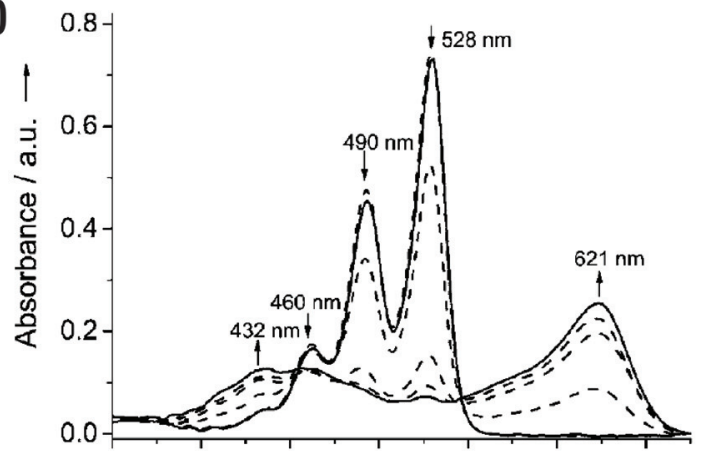

d)

e)
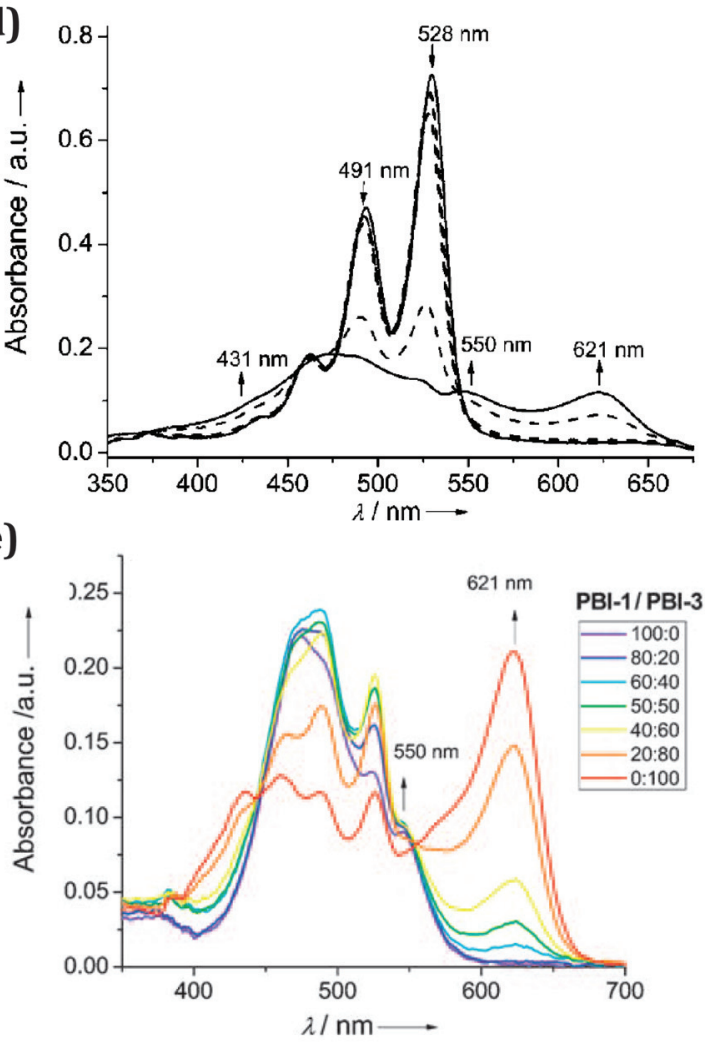

Figure 4 (a) Molecular structure of PBI 1-7. Solvent-dependent (in various $\mathrm{MCH} / \mathrm{CHCl}_{3}$ mixtures) UV/Vis absorption spectra of (b) PBI-1 (50:50 to 80:20), (c) PBI-4 (40:60 to 70:30), and (d) PBI-7 (50:50 to 90:10) at a concentration of $1 \times 10^{-5} \mathrm{M}$ at $25{ }^{\circ} \mathrm{C}$. Arrows indicate the spectral changes upon increasing the amount of $\mathrm{MCH}$ (from 40 to $90 \%$ ). (e) UV/Vis absorption spectra of a mixture of PBI-1 and PBI-3 in different ratios in 80:20 MCH/CHCl3 at $25^{\circ} \mathrm{C}$. The total concentration of chromophores remains constant $\left(1 \times 10^{-5} \mathrm{M}\right)$ in each mixture. Adapted with permission from Ref. 51 . Copyright 2008 John Wiley and Sons. 
peripheral side chains dictated the self-assembly mode, going from the usually found H-type to the J-type aggregates. PBIs containing linear alkyl side chains, which are sterically less demanding, formed face-to-face H-type aggregates (Figure 4b), while the PBIs having branched alkyl tails formed slipped J-aggregates (Figure 4c,d) or did not aggregate. The self-assembly studies were performed using mixtures of good and bad solvents: chloroform and methyl cyclohexane $(\mathrm{MCH})$, respectively. When the amount of $\mathrm{MCH}$ increased, the signature for the $\mathrm{H}$ - or J-type aggregates increased as well. This work also shows mixtures of PBIs prone to form $\mathrm{H}$ - or J-aggregates, for example, PBI-1 and PBI3 (Figure 4e). In this case, co-aggregation in H-type stacks with alternate packing of both chromophores was observed in the presence of a large amount of $\mathrm{H}$-aggregating $\mathrm{PBI}-1$. On the other hand, self-sorting of PBI-1 and PBI-3 was observed in the presence of a high content of J-aggregate-forming PBI3. These results are very relevant to help in the design of photovoltaic devices, for example, where highly organized arrays of donor and acceptor materials are needed. More recently, Wagner et al. have shown a similar PBI system modified with 1,7-dimethoxy substituents in the bay positions. ${ }^{52}$ The authors used this PBI derivative to study living supramolecular polymerization, achieving fluorescent J-type aggregates. In previous works, the authors used similar chemical designs without modifying the bay area. $^{53,54}$ The modifications on the bay area result in slightly core-twisted PBIs that self-assemble into metastable H-aggregates, which are transformed into thermodynamically stable fluorescent J-aggregates by seed-induced living supramolecular polymerization. The control over the supramolecular polymerization is very interesting for the design of functional supramolecular polymers and to study charge carrier transport processes.

Lochbrunner's group has reported several works on amide-containing PBI derivatives forming J-type aggregates, demonstrating their excellent exciton migration properties. In a work from 2011,55 this group explored the time dependence of the annihilation dynamics to characterize the mobility of singlet excitons in J-aggregates in tetraphenoxy-substituted PBIs. The authors observed by infrared spectroscopy that intermolecular $\mathrm{H}$-bonding between the pending amide groups stabilizes the aggregates in apolar solvents, forming bundles of long aspect-ratio aggregates. The delocalization length and the mobility of excitons in such aggregates were studied using femtosecond pumpprobe absorption spectroscopy. It was observed that excitons could not migrate in all three directions but that their mobility was anisotropic. A diffusion constant of $1.29 \mathrm{~nm}^{2} / \mathrm{ps}$ was found, corresponding to a maximal exciton diffusion length of $96 \mathrm{~nm}$ for the measured exciton lifetime of 3.6 ns. These results highlight the potential of such derivatives in optoelectronic devices and artificial $\mathrm{LH}$ systems due to their capacity to direct energy transport.
In 2012, the same group reported the size-dependent exciton dynamics in 1D self-assembled PBI derivatives. ${ }^{56,57}$ Tetraphenoxy-substituted H-bonded PBI assemblies were again investigated by ultrafast transient absorption spectroscopy and Monte-Carlo simulations as a function of temperature and the excitation density. At low temperatures the aggregates were considered as infinite chains, since the dynamics were dominated by diffusion-driven exciton-exciton annihilation. Upon increasing the temperature, the size of the aggregates decreases, consisting of only a few monomers. The authors observed a 1D diffusion length of $80 \mathrm{~nm}$ in long PBI chains, corresponding to $170 \mathrm{PBI}$ molecules. Variable concentration absorption and temperature-dependent fluorescence studies on the same tetraphenoxy-substituted $\mathrm{H}$-bonded $\mathrm{PBI}$ showed a biphasic aggregation behavior of that derivative. ${ }^{58}$ Long aggregates were formed at high concentration and low temperature, displaying J-type aggregation, while at intermediate concentrations and temperatures, dimers were found with $\mathrm{H}-$ type excitonic coupling.

Not only PBI derivatives functionalized with amide bonds have been used to study seeded polymerization where J-type aggregates play an important role. Very recently, Ogi et al. ${ }^{59}$ have reported a study of seeded polymerization in aqueous media of a DPP derivative with pending amide functionalities from the lactam rings. The authors managed to control the polymerization and the morphology of the aggregates, finding 1D fibers exhibiting J-type aggregation.

\section{Peptide-Chromophore Systems}

Oligopeptides, containing several amide bonds, are versatile building blocks to program organized structures of $\pi$-conjugated molecules. ${ }^{60}$ The study of natural and artificial self-assembled peptide structures has provided some design rules that allow the control on the morphology and function of materials with interesting electronic properties. $^{61}$ Together with the peptide sequences, the secondary structures play a major role in the final functions. Furthermore, there are systems described where the $\pi$-conjugated segments are embedded within the oligopeptide structure and oligopeptide systems terminally functionalized with the $\pi$-conjugated molecules in case the peptides are covalently linked to the chromophores. For instance, Sun et al. ${ }^{62}$ reported an oligopeptide of sequence Leu-Leu-Lys-Lys functionalized with anthracene as the $\pi$ conjugated part (Figure $5 \mathrm{a}$ ). This amino acid sequence was chosen due to its known $\beta$-sheet forming tendency that guides the formation of $1 \mathrm{D}$ structures. Anthracene was selected because it is known to form anisotropic assemblies via $\pi$-stacking. Self-assembled structures were achieved by casting saturated benzene solutions of the oligopeptide on silicon substrates. 1D fiber-like nanostructures were 


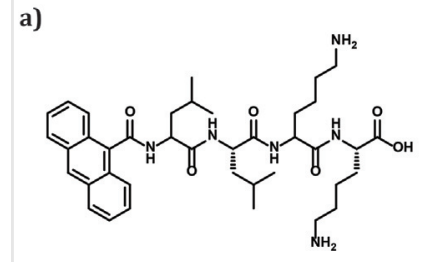

b)

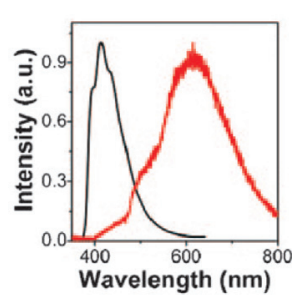

c)

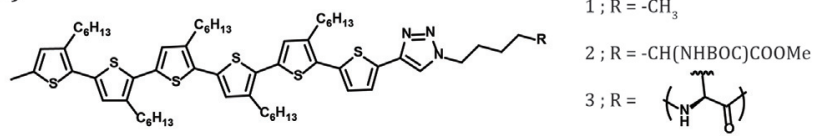

Figure 5 (a) Chemical structure of the LLKK-anthracene derivative. (b) Emission spectra of the Leu-Leu-Lys-Lys-anthracene derivative in an aqueous solution (black) and as 1D, self-assembled structures (red, the assembled structures were measured as solid with a confocal microscope). (c) Chemical structure of the L-lysine-sexithiophene derivative. Images (a) and (b) have been adapted with permission from Ref. 62. Copyright 2011 John Wiley and Sons. Image (c) has been adapted with permission from Ref. 63. Copyright 2011 American Chemical Society.

observed with microscopy techniques and subsequently studied by spectroscopy. The oligopeptide-anthracene in the solid state presented red-shifted emission (Figure $5 \mathrm{~b}$ ) and a shorter excited-state lifetime with respect to the oligopeptide-anthracene in water solution. These results confirm the presence of J-type aggregates between the anthracene dyes guided by H-bonding, enhancing electronic communication. The authors fabricated nanoelectronic devices to provide a direct conductivity measurement, achieving conductivity values several orders of magnitude higher (ranging from 0.011 to $0.107 \mathrm{~S} \mathrm{~cm}^{-1}$ ) than in bulk anthracene $\left(10^{-10} \mathrm{~S} \mathrm{~cm}^{-1}\right)$. Kumar et al. ${ }^{63}$ have shown a sexithiophene-electroactive segment attached to the side chain of L-lysine that was subsequently polymerized (Figure 5c). The self-assembly properties of the system were studied as well as the optoelectronic properties and the fabrication of photovoltaic devices and OFETs. The results were compared with a protected amino acid and an achiral nonpolymerized derivative (Figure 5c). Thin films were cast from solutions of the three derivatives and they all showed red-shifted absorption and formed J-type aggregates. The polymerized derivative forms an $\alpha$ helix secondary structure, while the control molecules do not form any helical structure and do not display any circular dichroism (CD) signals. Organic solar cells and OFETs were fabricated with the three derivatives, finding that the oligopeptide forming an $\alpha$-helix showed enhanced efficiency in solar cells reaching a power conversion efficiency of $0.22 \%$ versus 0.14 and $0.12 \%$ for the achiral sexithiophene and the protected amino acid, respectively. Furthermore, the polymerized derivative was the only compound among the three presenting hole mobilities $\left(1.9 \times 10^{-7} \mathrm{~cm}^{2} \mathrm{~V}^{-1} \mathrm{~s}^{-1}\right)$. The control molecules presented electron mobility with values of only $7.4 \times 10^{-4}$ and $2.8 \times 10^{-5} \mathrm{~cm}^{2} \mathrm{~V}^{-1} \mathrm{~s}^{-1}$ for the achiral sexithiophene and the protected amino acid, respectively. The results point out that the assembly of $\alpha$-helically templated chromophores leads to interconnected networks for charge transport. Other works have used peptides to direct the assembly of porphyrins. For example, Jintoku et al. ${ }^{64}$ have found that pyridylated $C_{60}$ interacts with zinc porphyrins functionalized with L-glutamide when they are assembled in J-aggregates. The interaction between the porphyrin and the modified $\mathrm{C}_{60}$ changes the morphology of the porphyrin's assemblies from entangled fibers to vesicles.

Apart from covalently coupling peptides to chromophores, noncovalent approaches where the peptides assemblies guide the organization of chromophores have been pursued. For instance, Koti and Periasamy ${ }^{65}$ have shown the J-aggregation of TPPS guided by polylysine. The concentration of lysine residues needed to induce J-aggregate formation was demonstrated to be 3 orders of magnitude lower than that when monomeric lysine was used. In this case, chirality is transferred from the polypeptide to the porphyrin complex, which is achiral, achieving rod-like structures with J-aggregation of several hundreds of nanometers.

Short peptides have also been studied to mimic LH complexes. Park's group has sown nanotube-forming peptides based on L-Phe-L-Phe (FF) systems ${ }^{66}$ that incorporate hydrophobic porphyrin chromophores in the nanotubes. The optical spectra showed that the porphyrin dyes were assembled into J-aggregates, resulting in strong exciton coupling between the porphyrin monomers. Apart from porphyrin derivatives, ruthenium chromophores have been successfully immobilized on the surfaces of $\mathrm{FF}$ nanotubes and derivatives of FF peptides, such as FmocFF. Fmoc-FF forms hydrogels with a $\beta$-sheet secondary structure that is stable after co-assembly with either ruthenium complexes or porphyrin derivatives. In all cases, the incorporated chromophores self-assemble into large Jtype aggregates with strong exciton coupling. ${ }^{67-69}$

\section{Urea-Containing Systems}

Together with amides, urea groups are the most common $\mathrm{H}$-bonding motifs that have been incorporated in multiple dyes to study their self-assembly properties. $^{70-73}$ Das and Ghosh ${ }^{74}$ studied the differences between amide and urea $\mathrm{H}$-bonding motifs within the same $\pi$ conjugated system. In this work, the authors tried to give an answer to whether the mismatch in number of $\mathrm{H}$-bonding interaction sites would influence the stability of the selfassembled structures or if it would alter the chromophoric arrangement. For this purpose, they synthesized two 
a)

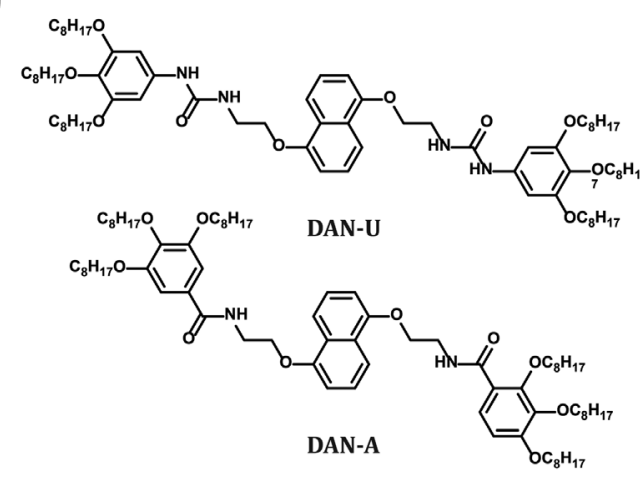

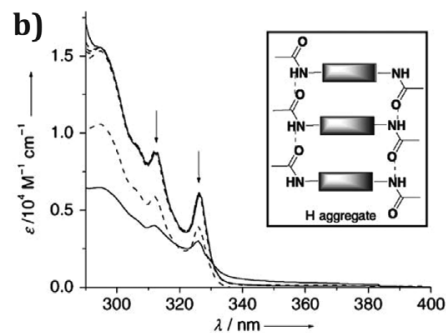

c)

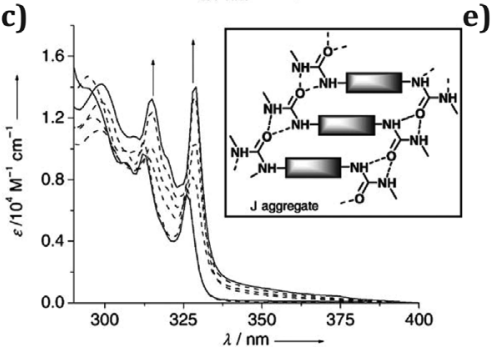

d)

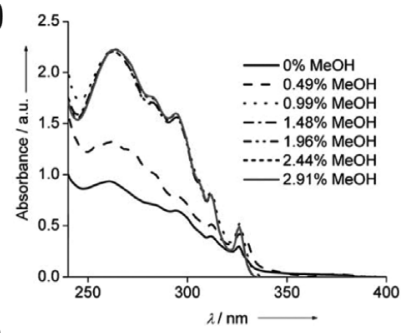

e)

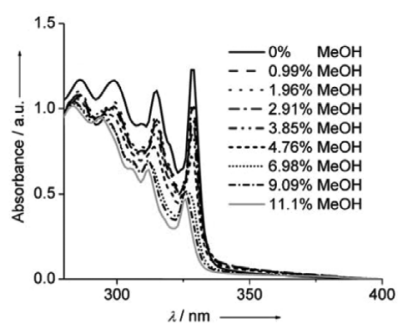

Figure 6 (a) Chemical structures of DNA-U and DAN-A. Solvent-dependent absorption spectra of DAN-A (b) and DAN-U (c) Arrows indicate direction of spectral changes from THF to MCH. Inset: schematic representation of the proposed mode of chromophore assembly (hydrogen bonding is shown with a dashed bond). Concentration of the chromophore $=0.1 \mathrm{mM}$ and temperature $=25^{\circ} \mathrm{C}$. Effect of gradual addition of MeOH on the absorption spectra of the self-assembled structure of DAN-A (d) and DAN-U (e) in $95 \% \mathrm{MCH} / \mathrm{THF}$. Concentration $=0.1 \mathrm{mM}$, Temperature $=25^{\circ} \mathrm{C}$. Reprinted with permission from Ref. 74. Copyright 2010 John Wiley and Sons.

derivatives of the dialkoxynaphthalene (DAN) $\pi$-system, namely, DAN-U and DAN-A (Figure 6a), which differ only in amide and urea functionalities. The authors observed that DAN-U formed J-aggregates in solution with a superior selfassembly propensity and thermal stability than the DAN-A system, which formed H-type aggregates. UV/Vis spectroscopy in solution showed the differences in aggregation at the same concentration and equal solvent (Figure $6 b, c$ ). To demonstrate the strength of the J-aggregates, absorption spectra were recorded after the addition of progressive amounts of a H-bonding competing solvent (methanol; Figure 6d,e). The authors observed that for DAN-U the amount of methanol needed to disrupt the Jaggregate band and recover the monomeric state spectrum was larger (11\%) than that for DAN-A (less than 3\%). The differences in self-assembly behavior also resulted in different gelation properties for the two systems. The urea-functionalized system showed gelation in a wide variety of organic solvents with higher thermal stability and lower critical gel concentration, whereas the amidefunctionalized derivative formed gels with higher mechanical stability. These differences were explained by comparing the rigidity of the fibers formed by the gels. These findings are very relevant since they offer valuable information for the design of $\mathrm{H}$-bonded semiconductors and the desired architectures they might form. Other ureacontaining $\pi$-conjugated systems have been reported by Liu et $a .^{75}$ They reported a monopyrrolotetrathiafulvalenebased derivative containing a urea group and its gelation properties were explored. The urea TTF derivative was proven to be a nongelator in single solvents, but it did gel mixtures of chloroform/dichloromethane and $n$-hexane. Fourier transform infrared (FTIR) spectroscopy, UV/Vis absorption spectroscopy, and SAXS (small-angle X-ray scattering) revealed that in the organogel system, the gelators self-assembled into supramolecular networks with a J-type aggregation mode under the joint effect of $\pi-\pi$ stacking, intermolecular $\mathrm{H}$-bonding, and van der Waals forces. The gel morphology obtained, thanks to the Jaggregation mode of the TTF-urea derivative, provided the gel with great adsorption properties for other fluorescent dyes. Furthermore, the gel responded to external stimuli, making this assembly approach very appealing for the design of smart soft materials.

Urea $\mathrm{H}$-bonding motifs have also been incorporated in $\mathrm{BChl}$ analogues, forming lamellar supramolecular structures exhibiting J-aggregation. ${ }^{76}$ In the work of Shoji et al. there is a specific study on the effect of H-bonding in chlorosomal assemblies. Zinc BChl-d analogues with urea and amide $\mathrm{H}-$ bonding groups self-assembled in hexane solutions forming chlorosomal J-aggregates. The authors performed spectroscopic analysis of the $\mathrm{BChl}$ analogues and found that the formation of J-aggregates was assisted additionally by $\mathrm{H}-$ bonding. Microscopy analysis showed that the additional $\mathrm{H}-$ bonding units guided the assemblies into highly ordered supramolecular structures even in comparison to the tubular structures formed by BChl-d. The same group has also reported artificial chlorosomal supramolecular nanosheets formed by the self-assembly of a zinc methoxychlorophyll derivative bearing amide and urea groups at the 
17th position. ${ }^{77}$ In this case, the zinc chlorophyll kinetically formed dimeric species that were transformed into thermodynamically more stable chlorosomal J-aggregates in the solid state. Once again, this was possible thanks to the additional H-bonding motifs pending from the main framework. The kinetic product had particle-like nanoassemblies, while the thermodynamic product presented sheet-like nanostructures, which is the first case of biomimetic supramolecular nanosheets of chlorosomal Jaggregates obtained from synthetic zinc chlorophyll derivatives with additional urea moieties. This molecular design offers several applications in artificial LH antennas and photosynthesis-inspired processes. Some of these studies also presented the ester-containing analogues as control molecules, not showing the same supramolecular structures and strongly coupled J-aggregates, highlighting the important role of having directional H-bonding groups. ${ }^{76}$

\section{Systems Containing Complementary H-Bonds}

The arrangement of different $\pi$-conjugated components via complementary H-bonding is a great strategy towards highly organized structures with enhanced optoelectronic properties.
Initial works by Schenning et al. ${ }^{11}$ in 2002 showed how $\pi$-conjugated systems with complementary H-bonding motifs resulted in PET in donor-acceptor dyads (Figure 7a). The authors report the collective and hierarchical self-assembly of oligo( $p$-phenylene vinylene) (OPV) as the donor material and a PBI derivative as the acceptor into chiral fibers. The PBI derivative has two complementary $\mathrm{H}-$ bonding binding sites for the diaminotriazine OPV (Figure 7a). The binding constant for this donor-acceptordonor (D-A-D) system was low in chloroform, which is a good solvent, but it increased significantly in more apolar solvents, like $\mathrm{MCH}$. The authors studied the self-assembly process of the D-A-D system via UV/Vis, fluorescence, and $\mathrm{CD}$ titration experiments. Regarding the absorption of the PBI component, a red-shift of $\lambda_{\max }$ from 562 to $604 \mathrm{~nm}$ was observed, which is much larger than what is observed in PBI aggregates. The authors attribute such a red-shift to the tight packing of dyes in a J-type aggregate. In this case, there is fluorescence quenching, rationalized by electron transfer from the OPV component to the PBI. They observed that optimal quenching happened when all PBI binding sites were $\mathrm{H}$-bonded to the OPV in a 1:2 ratio. Therefore, the authors suggested the H-bonded structure shown in Figure 7a. Furthermore, a strong negative Cotton effect of the PBI was observed when adding progressively OPV

a)

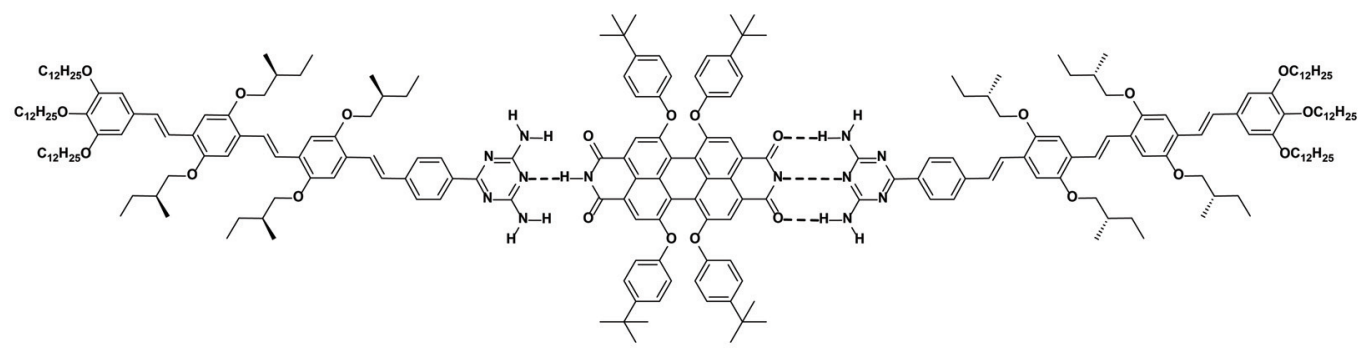

OPV PBI OPV

b)

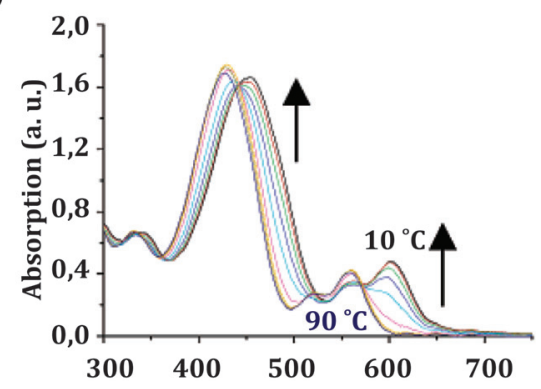

c)

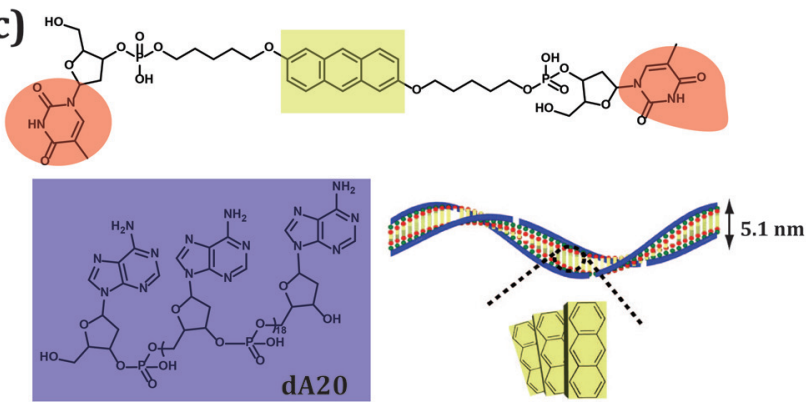

Figure 7 (a) Molecular structures of the OPV and PBI derivatives and their H-bonded assembly. (b) Temperature dependent UV/Vis of the D-A-D triad. (c) Chemical structure of the anthracene derivative coupled to DNA bases and schematic representation of the templated structure formed. Concentration $=3.7 \times 10^{-5} \mathrm{~mol} / \mathrm{L}$ ) in $\mathrm{MCH}$ (arrows indicate the changes upon cooling). Reprinted with permission from Ref. 11 . Copyright 2002 American Chemical Society. 
chromophore, indicating the transfer of chirality of the OPV side chains to the PBI component. Similar results were found when the concentration of OPV was constant and PBI was added. With these optical activity results, the authors provided clear evidence that the D-A-D complex stacks into J-aggregates with a helical screw sense. Variable temperature spectroscopy was also carried out to complete the selfassembly study (Figure 7b). At high temperature (higher than $60{ }^{\circ} \mathrm{C}$ ), the absorption spectra resemble the ones of the separate compounds in $\mathrm{MCH}$ at room temperature. Together with these changes in UV/Vis, the $C D$ signal for both components is lower at temperature above $40{ }^{\circ} \mathrm{C}$ and completely disappeared at $60{ }^{\circ} \mathrm{C}$. Upon cooling, the $\mathrm{CD}$ spectrum fully recovered, indicating reversibility. Additionally, it was observed that the photoluminescence of the PBI increased at high temperature, when the system was disassembled. The three techniques indicated the presence of aggregates at low temperature and molecularly dissolved monomeric species at high temperature, with a melting temperature of approximately $50{ }^{\circ} \mathrm{C}$. Femtosecond pumppulse spectroscopy measurements were performed in aggregated solutions to determine the charge transfer rate in the D-A-D system. When the D molecule is excited, the OPV radical cation becomes visible at $1450 \mathrm{~nm}$, indicating a PET process. At room temperature this charge-separated state is formed within a few picoseconds (ps), while the recombination happens within $60 \mathrm{ps.} \mathrm{At} 80^{\circ}$ $\mathrm{C}$ where only monomers and small $\mathrm{H}$-bonded complexes are present, the charge formation happens again within ps, but the recombination becomes slower probably because after charge separation, the D-A-D complexes dissociate. With this work, the authors demonstrated that the organization of $\mathrm{p}-\mathrm{n}$ heterojunctions was possible and that electronic processes could be controlled.

Other examples, like the work reported by Kar et al., 78 have shown a D-A-D core-substituted naphthalene-diimide (cNDI) chromophore that exhibits highly cooperative Jaggregation leading to nanotubular assemblies and gelation in organic solvents. It was the first report of a H-bondinginitiated supramolecular polymerization of a diaminosubstituted cNDI (NDI-H; Figure 8). ${ }^{78}$ The authors showed that the final tubular assembly enables very effective delocalization of excited states resulting in very long excited-state lifetimes. They studied the supramolecular polymerization of NDI-H on a selection of organic solvents and they noted that in the presence of a D-A-D H-bonding competitor, gelation was prevented (Figure $8 \mathrm{a}$ ). This was attributed to the NDI-H being endowed with a D-A-D type complementary H-bonding motif, indicating that supramolecular polymerization by extended $\mathrm{H}$-bonded chain formation among the imide groups of the NDI-H is essential for gelation to occur. UV/Vis spectroscopy showed pronounced a)

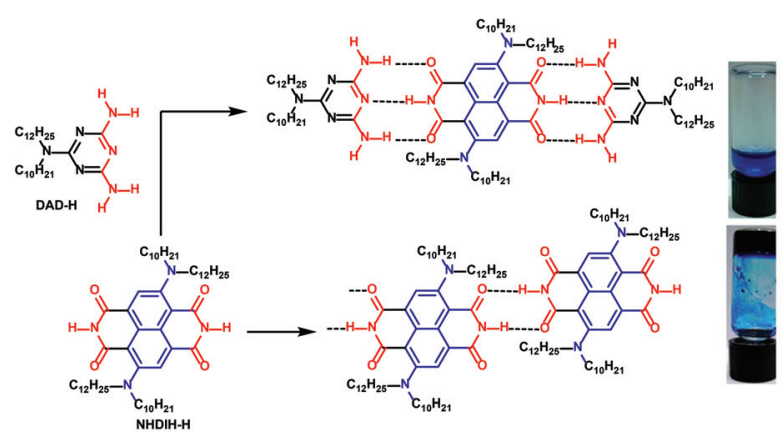

b)

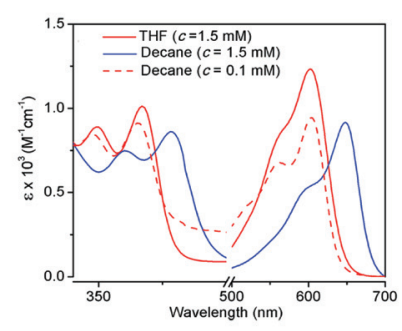

c)

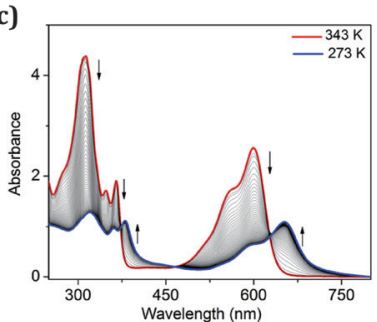

d)

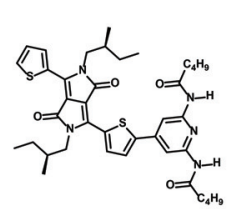

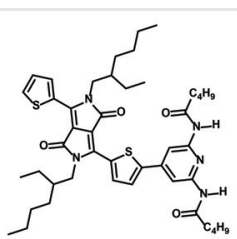

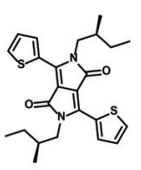

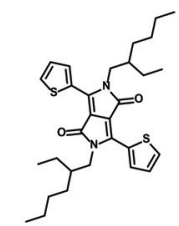

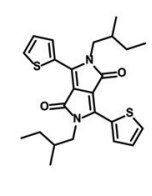

e)

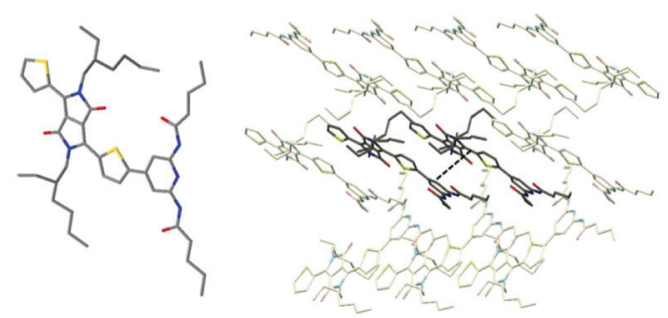

Figure 8 (a) H-bond driven supramolecular polymerization and gelation (right, bottom) of NDI-H in n-decane ( $c=4.0 \mathrm{mM}$ ). The addition of a D-A-D Hbonding competitor (2.0 equiv.) prevents the gelation (right, top). (b) Solvent- and concentration-dependent UV/Vis spectra (intensity normalized with concentration) of NDI-H $(I=0.1 \mathrm{~cm}, T=298 \mathrm{~K})$. (c) Temperature-dependent UV/Vis experiments of NDI-H (1.75 mM, decane). Arrows indicate the spectral changes upon decreasing the temperature. (d) Molecular structures of the DPP derivatives studied. (e) Single-crystal structure of the DPP derivative modified with diamidopyridine. Images (a), (b), and (c) have been reprinted with permission from Ref. 78. Copyright 2016 Royal Chemical Society. Images (d) and (e) have been reprinted with permission from Ref. 28. Copyright 2016 John Wiley and Sons. 
solvent effects, finding monomeric features in THF and a large bathochromic shift for the $\pi-\pi^{*}$ band in $n$-decane, indicating J-aggregation. Additionally, the intramolecular charge transfer band presented a bathochromic shift as well, indicating the reduction of the HOMO-LUMO gap due to effective delocalization of the charge transfer state in Jaggregates (Figure 8b). UV/Vis temperature-dependent studies were carried out in n-decane at different concentrations to further analyze the J-aggregate (Figure 8c). They observed that upon cooling, depletion of the absorption maxima at multiple wavelengths occurred at the expense of new red-shifted transitions, which were attributed to Jaggregation. The appearance of isosbestic points also revealed the thermodynamic equilibrium between monomeric and self-assembled species. When the fraction of aggregated species at different concentrations was plotted against temperature, sharp nonsigmoidal curves were obtained, thus indicating cooperative self-assembly. They proposed that NDI-H initially undergoes a linear oligomerization via $\mathrm{H}$-bonding and when the length of the oligomer becomes sufficiently large, J-aggregation and alkyl chain packing become predominant, leading to the formation of a $2 \mathrm{D}$ sheet that eventually bends to generate nanotubes. They concluded that J-aggregated dye molecules encapsulated in the multilayer walls of the tubes facilitate very effective delocalization of the excited states, yielding remarkably prolonged excited-state lifetimes.

Self-complementary H-bonding motifs have been widely explored by Yagai et al. ${ }^{79}$ They have shown multiple examples of supramolecular polymers based on H-bonding interactions between naphthalene-based molecules containing barbituric acid. Earlier works in $2012^{80}$ showed how regioisomers (substitution at positions 2,6 or 1,4 ) of naphthalene-barbiturate derivatives resulted in different types of topologies in the supramolecular polymers obtained. The presence of the barbiturate results in the formation of $\mathrm{H}$-bonded rosettes composed of six naphthalene units. The authors observed that the 2,6-substituted naphthalene formed nanorings, while the 1,4-substituted analogue resulted in nanorods. Such rosettes have different geometrical arrangements that favor either J- or H-excitonic coupling of the naphthalene cores for the nanorings and the nanorods, respectively. The authors demonstrated that the regioisomeric introduction of barbituric acid provides control over the self-assembled structures. Subsequently, more expanded $\pi$-conjugated systems based on naphthalene-barbiturate derivatives were explored by the same group. In this case, they introduced azobenzene moieties into the 2,6 or 1,4 -substituted naphthalene-barbiturate ${ }^{81}$ derivatives and explored the folding-unfolding properties of the supramolecular polymers using light as an external stimulus.

Work inspired by the complementary H-bonding between DNA bases has rendered very interesting examples in the formation of helical J-aggregates. For instance, Iwaura et al. $^{82}$ have shown the templating effect of oligoadenylic acid (dA20) to arrange anthracene dyes functionalized with thymidylic acid (Figure 7c). The authors found the formation of J-aggregates in the dA20 templated systems using UV/Vis, fluorescence, $C D$, and atomic force microscopy. Interestingly, such types of aggregates were not found when only the anthracene-thymidylic acid was studied. They proposed a dye arrangement (Figure 7c), where the A-T base-pair formation induces a head-to-tail arrangement of the transition moments along the anthracene short axis in the templated assemblies.

DNA base pairs have also been recently reported to study energy transfer in J-aggregates of PIC dyes. ${ }^{83-85}$ For instance, Mandal et al..$^{83}$ have studied the excitonic properties of PIC Jaggregates using poly(dA)-poly(dT) of different lengths. Furthermore, they have studied energy transfer from a quantum dot as the donor to the dye Alexa Fluor (AF647) as the acceptor through a bridge of J-aggregated PIC dyes templated by DNA. The authors observed significant energy transfer over longer distances than the ones possible without the PIC J-aggregate bridge. In a different set of experiments the authors introduced a GC (guanosinecytosine) discontinuity within the dA-dT strand, observing a decrease in the efficiency of energy transfer. This result highlights the importance of having a continuous Jaggregate to enhance energy transfer.

Markova et al. ${ }^{86}$ have demonstrated the use of cyanine dyes as chiroptical reporters (probes for structural determination) in oligonucleotide-cyanine conjugates. Such conjugates show high susceptibility to monomer-to-dimer switches due to the formation of J-aggregates, which display intense CD signals in the DNA duplex.

Different complementary H-bonding motifs have been recently studied by Zhou et al. ${ }^{87}$ They show how the conjugation length, solubilizing side chains, and the type of H-bonding groups affect the homo-assembly of DPPs into Jaggregates. $^{28}$ These measurements were performed through temperature-dependent UV/Vis titrations in toluene on multiple thiophene DPP derivatives (Figure 8d,e). They found out that the presence of diamidopyridine (DAP) groups, known for their $\mathrm{H}$-bonding ability, improved considerably the driving force for assembly into larger clusters compared to those without the DAP group. These findings described the intricate contributions from $\mathrm{H}$ bonding, $\pi-\pi$ stacking, van der Waals forces, solvent, concentration, and temperature are responsible for the size, structure, stability, and spectroscopic attributes of the resulting superstructures. Furthermore, their main finding was that these molecules assemble into J-aggregates, since they obtained crystalline structures where the molecules appeared in slip-stack geometries (Figure 8e). The authors derived two equations for the calculation of the size of an aggregated stack and its average mole fraction. These 
discoveries are very relevant, since the structure and properties of supramolecular assemblies are intimately linked, impacting the design of devices. Furthermore, the authors have explored complementary H-bonded DPPs and $\mathrm{PBI} / \mathrm{NDI}$ systems, finding interesting effects on the excitedstate dynamics and on the activation of new pathways, such as singlet fission. By making combinations of complementary H-bonded DPPs and PBIs/NDIs, the authors observed differences in the molecular packing of the aggregates and could identify the best combination to achieve triplet quantum yields as high as $65 \%$ for a correlated pair of triplets and $15 \%$ for uncorrelated pair of triplets. ${ }^{88}$

\section{Systems with Other H-Bonding Functionalities}

Examples of molecules containing hydroxyl functional groups and displaying J-aggregate formation have also been reported. For instance, Villari et al. ${ }^{89}$ have compared two porphyrin derivatives (P-trans-OH and P-trans-Et). P-trans$\mathrm{OH}$ contains free hydroxyl groups at the periphery, while $\mathrm{P}$ trans-Et has ethoxy groups (Figure 9a). Both derivatives form large aggregates, but P-trans- $\mathrm{OH}$ forms J-type aggregates, as evidenced by the red-shifted main extinction band (Figure 9b) and a shortening of the fluorescence lifetime. On the other hand, the substitution of $-\mathrm{OH}$ by $\mathrm{OCH}_{2} \mathrm{CH}_{3}$ results in the formation of $\mathrm{H}$-type aggregates, which appeared as a blue-shifted transition in the UV/Vis spectrum and the long fluorescence lifetime. The results obtained with different spectroscopy techniques showed that H-bonding guides the assemblies to form J-aggregates. The measurements were performed in aqueous solutions, achieving aggregation through $\mathrm{H}$-bonding and avoiding competition with the water molecules. This result is important to design systems that mimic natural systems and that can be applied in bioelectronic devices.

Our group has recently shown H-bonded thiophenecapped DPP derivatives functionalized with semicarbazone groups displaying J-aggregates ${ }^{90}$ that had different intensities depending on the solvent used. While chloroform solutions did not show any types of aggregates, chlorobenzene and toluene solutions showed an additional redshifted band that increased dramatically in ethyl acetate. FTIR spectroscopy in solution showed the formation of $\mathrm{H}$ bonds and we confirmed that the J-aggregates were formed by $\mathrm{H}$-bonding interactions by adding a H-bonding competing solvent (methanol). The J-aggregate band totally disappeared after the addition of specific amounts of methanol. J-aggregates were also present in solutions of self-assembly promoting solvents at room temperature and they shaded upon increasing the temperature. Nevertheless, J-aggregates were still observed at high temperatures (up to $60{ }^{\circ} \mathrm{C}$ ), highlighting the strength of the assemblies. With this strategy, it was possible to tune the optoelectronic properties, such as the optical energy band gap or the coverage of the solar spectrum through aggregation.

Imide functional groups have also been exhaustively studied, particularly in PBIs. ${ }^{50}$ In 2017 Herbst et al. reported the achievement of an unprecedented organization of PBIs in a columnar liquid-crystalline (LC) phase, for which the transition dipole moments of the dye were oriented parallel a)

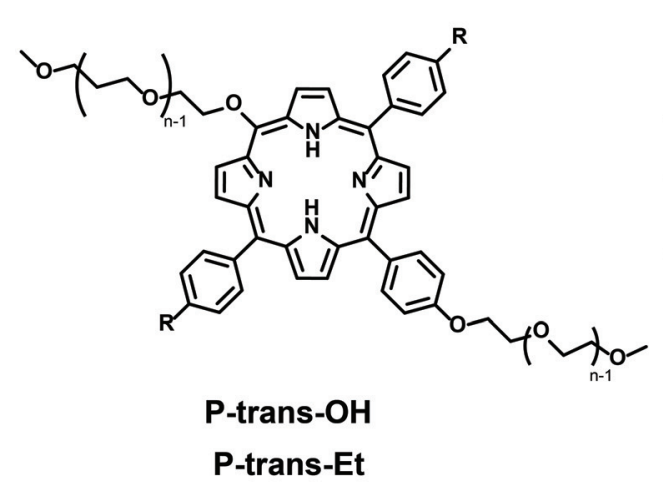

b)

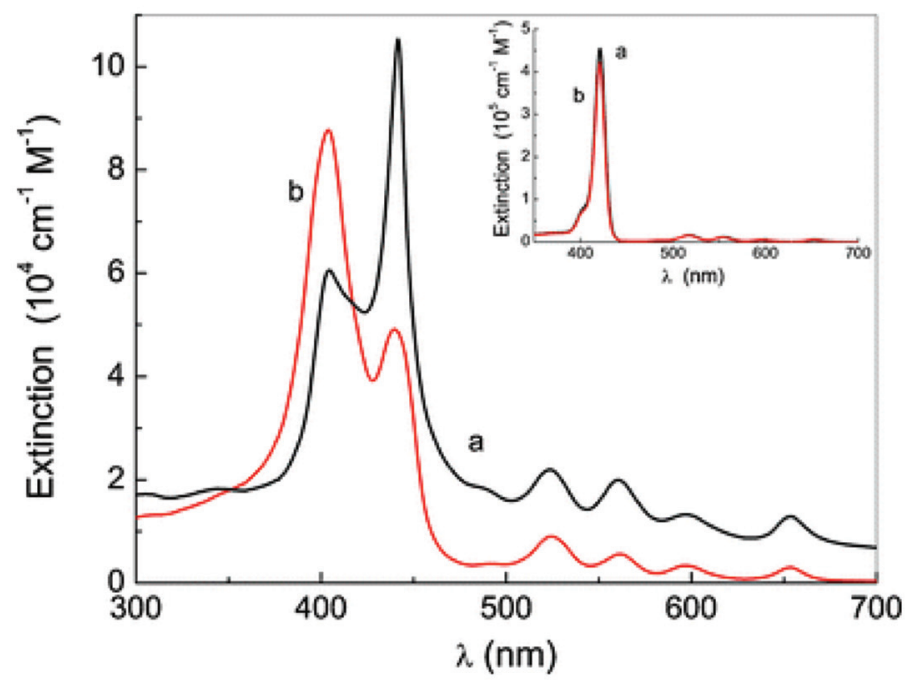

Figure 9 (a) Molecular structures of P-trans-OH and P-trans-Et. (b) Extinction spectra of P-trans-OH (curve a) and P-trans-Et (curve b) in water. The inset shows the corresponding absorption spectra in THF. Reprinted with permission from Ref. 89. Copyright 2012 Royal Chemical Society. 
a)

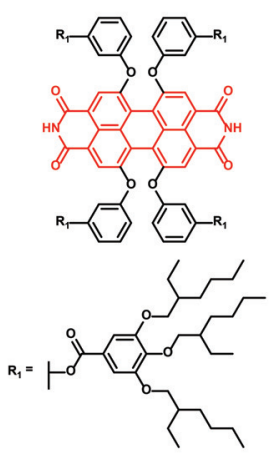

MEH-PBI

b)

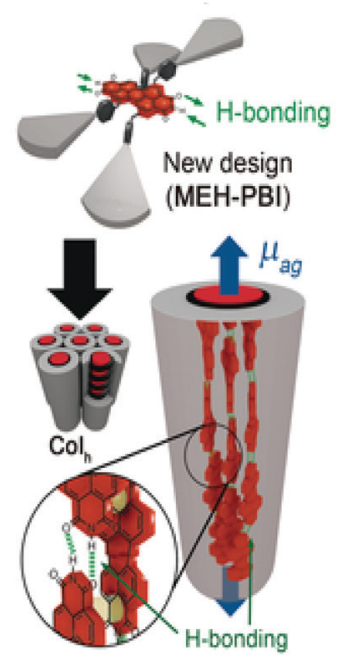

Strongly coupled J-aggregates

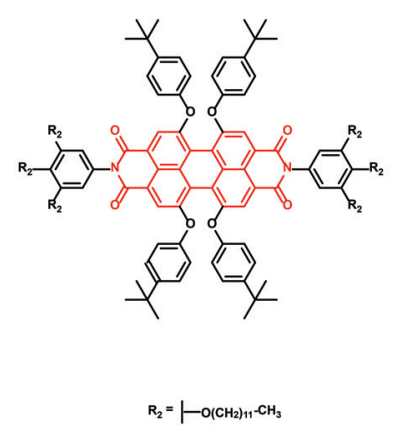

REF-PBI

c)

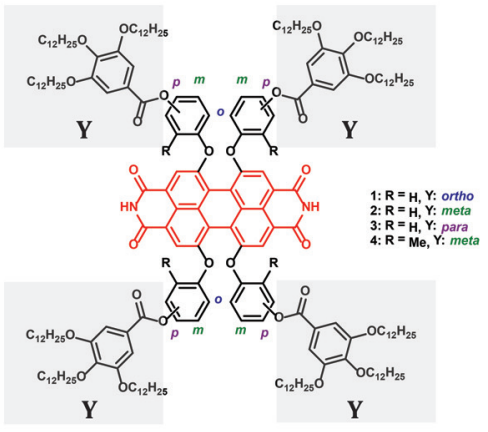

d)

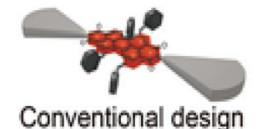

(REF-PBI)

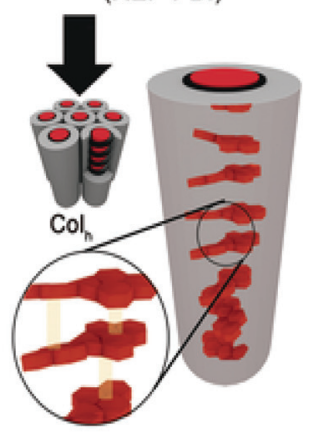

Weakly coupled aggregates

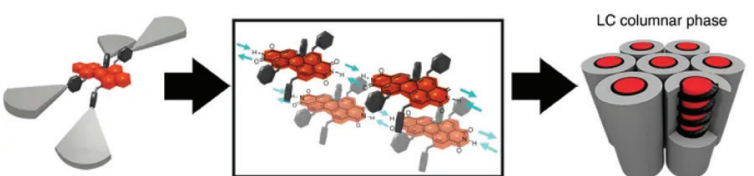

Multi-stranded PBI assemblies
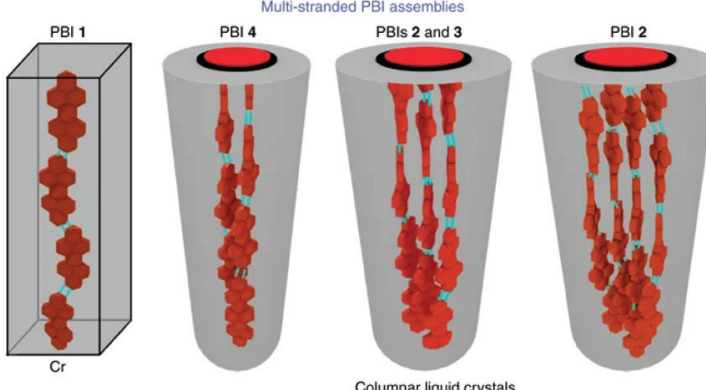

Columnar liquid crystals

Figure 10 (a) Molecular structures of MEH-PBI and reference molecule REF-PBI. (b) Illustration of the molecular self-assembly of MEH-PBI and REF-PBI into columnar hexagonal LC phases that exhibit orthogonal orientation of the PBIs. Blue arrows indicate the direction of the main transition dipole moments $\left(\mu_{\mathrm{ag}}\right)$ of the PBI molecules for MEH-PBI. (c) Chemical structures of PBIs 1-4. (d) Schematic illustration of the different columnar assemblies composed of one, two, three, and four strands. The helicity in the columnar assemblies of PBIs 2-4 has been chosen to be (P) for graphical representation. Please note that PBIs $1-4$ are racemic and therefore (P) and (M) helices may coexist in the corresponding columnar phases. Images (a) and (b) have been reprinted with permission from Ref. 91. Copyright 2017 John Wiley and Sons. Images (c) and (d) have been reprinted with permission from Ref. 92. Copyright 2018 Springer Nature.

to the columnar axes (Figure 10). ${ }^{91}$ This dye holds 2ethylhexyl-substituted gallic acid residues at the metapositions in a tetraphenoxy-functionalized perylene bisimide (MEH-PBI) possessing $\mathrm{N}-\mathrm{H}$ groups at the imide positions (Figure 10a) that self-assembles forming $\mathrm{J}$ aggregates through $\mathrm{H}$-bonds and $\pi-\pi$ interactions.

Usually, PBI liquid crystals are prepared by functionalizing the PBI core with mesogenic units at the imide positions, leading to the spontaneous stacking of the PBI cores through co-facial $\pi-\pi$ interactions forming columnar assemblies. This type of assembly was also reported for REFPBI (Figure 10a,b), leading to aggregates with a broad and slightly red-shifted absorption band and an LC columnar hexagonal phase. However, by bay tetrasubstituting PBIs with free NH groups at the imide positions, strongly coupled J-aggregates were obtained (Figure 10b). These J-aggregates were formed upon self-assembly in $\mathrm{MCH}$, which directed the formation of 1D helical structures with exciton migration over distances up to $100 \mathrm{~nm}^{39}$ Through a series of spectroscopy analysis and model simulations, they confirmed this unprecedented self-assembly arrangement for columnar LCs and established that its transition dipole moment is oriented parallel to the columnar axis, whereas for REF-PBI (as for other common discotic LCs), the orientation of the transition dipole moment is orthogonal to the column axis. These molecules are strongly coupled in 
1D J-aggregates and can be aligned in anisotropic thin films, opening up new perspectives in the field of photonic materials. In a more recent work, Herbst et al. ${ }^{92}$ continued studying the assembly mode for columnar PBI LCs analyzing the driving forces participating in the assembly process, and understanding how to control the number of strands in the columns via molecular engineering of the PBI building blocks. In this work, they reported how the molecular engineering of tetra-bay dendronized PBIs (PBIs 14, Figure 10c) can serve in the design of complex supramolecular assembly packing patterns, along with interesting optical properties. They described the selfassembly behavior of the PBI dyes self-organizing into one-, two-, three-, or four-stranded J-aggregates within crystalline and columnar LC phases (Figure 10d). They were able to tune the composition of these columnar assemblies through the molecular design guided by the corresponding dendron wedges; determining that the relative position of the dendrons at the phenoxy spacers of the PBI cores is the key factor that governs the respective packing structure via steric requirements, influencing parameters such as the helical pitch, the twist of the perylenes, and the number of strands in a single column.

The authors used a wide variety of spectroscopic techniques and theoretical models to analyze all the structural features of these columnar LCs. Spectroscopic investigations were made since the different organizations of PBIs 1-4 offer the opportunity for elucidating the influence of particular packing arrangements on functional properties of dye aggregates, especially in terms of exciton coupling. By comparing the optical properties in the bulk state to the ones of their monomers in solution through UV/Vis absorption spectra, a slight bathochromic shift with retained vibronic structure was observed for PBI 1, which supports that this compound does not pack in multiple strands due to the steric effects imparted by the orthosubstitution. In contrast, the other PBIs showed pronounced bathochromic shifts of $\lambda_{\max }$, which was strongest for double-stranded PBI $4\left(>2000 \mathrm{~cm}^{-1}\right)$ and decreased with increasing number of strands from PBI 3 $\left(>1800 \mathrm{~cm}^{-1}\right.$; three strands) to the quadruple-stranded PBI $2\left(>1700 \mathrm{~cm}^{-1}\right)$. All these structural features directly convert into functional properties due to the electronic coupling of the transition dipole moments of the closely stacked dyes, thus demonstrating that the bathochromic shift of the absorption maximum in the aggregated state relative to the monomer depends on the number of selfassembled strands and yields the best J-aggregate for the double-stranded arrangement of PBI 4, meaning that strongly coupled J-aggregates are promising materials for photonic applications. More recently Hecht et al. have synthesized a novel core-shell structured columnar LC, composed of a D-A dyad of tetraphenoxy PBI containing four bithiophene units at the periphery, PBI 2T a)

b)
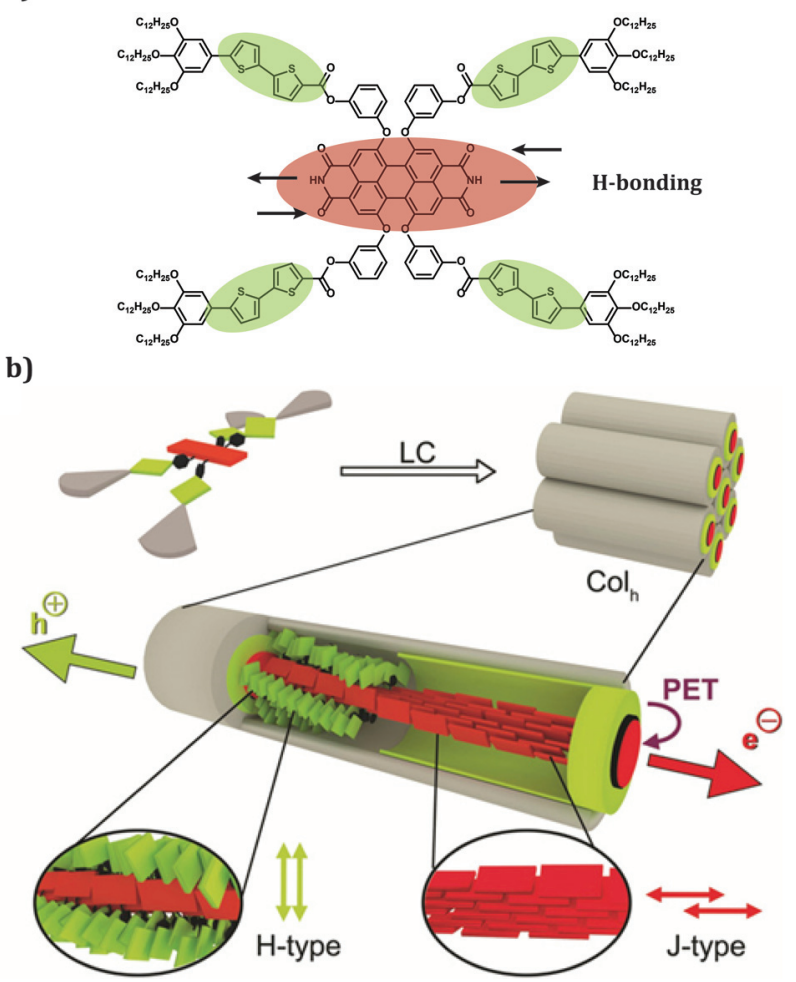

Figure 11 (a) Chemical structure of PBI 2T. (b) Illustration of the molecular arrangement of $\mathrm{PBI} 2 \mathrm{~T}$ in the columnar hexagonal $\mathrm{LC}$ phase with J-coupled PBls (red) and $\mathrm{H}$-coupled bithiophene units (green). The purple arrow indicates the photoinduced electron transfer (PET) and the consecutive electron and hole transport along the columnar core-shell structure. The helicity in the shown columnar assembly has been chosen to be $(\mathrm{P})$ for graphical representation. Please note that $\mathrm{PBI} 2 \mathrm{~T}$ is achiral and therefore $(P)$ and $(M)$ helices may coexist in the LC phase. Reprinted with permission from Ref. 93. Copyright 2019 John Wiley and Sons.

(Figure 11a). ${ }^{93}$ This molecule self-assembles in solution into helical J-aggregates guided by $\pi-\pi$ interactions and $\mathrm{H}$ bonds (Figure 11b), which organizes into an LC columnar hexagonal domain in the solid state. The properties of PBI 2T were studied by UV/Vis absorption and fluorescence spectroscopy, focusing on PET and energy transfer. An optical signature of monomerically dissolved tetraphenoxy-substituted PBIs was observed from the UV/Vis absorption spectrum of PBI $2 \mathrm{~T}$ in chloroform, with a prominent $\mathrm{S}_{0}-\mathrm{S}_{1}$ transition at $569 \mathrm{~nm}$.

An additional strong absorption band can be seen at $385 \mathrm{~nm}$, corresponding to the four electron-rich bithiophene side arms. Furthermore, these UV/Vis absorption spectra also unveiled the contrasting coupling behavior of the two chromophores within PBI 2T, and a model of the helical structure of PBI 2T was generated based on the experimental data. The PBIs were modeled to form a H-bonded sextuple-stranded helix with slipped-stack 
arranged chromophores. Theoretically, this sextuplestranded self-assembly mode should enable highly efficient exciton transport over long distances, as similar PBI J-aggregates with smaller number of strands, which have shown long exciton lifetimes, allow exciton transport over distances of roughly $70 \mathrm{~nm} .{ }^{39}$ The authors hypothesized that a higher number of strands in the core-shell structure of PBI 2T should improve its capability to avoid trap sites within the aggregate, therefore improving exciton mobility. This suggested that the material could be suitable for photoconducting applications and for this purpose, devices were fabricated by spin-casting a solution of PBI $2 \mathrm{~T}$ onto bottom contact $\mathrm{Si} / \mathrm{SiO}_{2}$ substrates with grid-like gold electrodes. The active layer in these devices showed a linear response to light intensity, with instant switching times within the measuring interval of the experiment. The benefits of the A-D core-shell architecture were notoriously evident when they compared these values to the ones obtained from devices that were fabricated with the parent compound from which PBI 2T was derived, which exhibited a quadruplestranded $14_{1}$ helix in the LC state. The interaction between PET and charge transport along the selfassembled core-shell structure allowed the implementation of the dye in two-contact photoconductivity devices, giving rise to a 20 -fold increased photoresponse compared to a reference dye without bithiophene donor moieties. ${ }^{92}$

\section{Conclusions and Outlook}

We have provided an overview on the most relevant and recent examples in the field of $\mathrm{H}$-bonded $\pi$-conjugated systems showing J-type aggregation. The additional $\mathrm{H}$ bonding moieties incorporated into the chemical structures of chromophores result in slipped-stack aggregates with strong exciton coupling. Such arrangement has been proven to be very beneficial for charge transport, making this supramolecular strategy very attractive in the field of organic electronics. Several hydrogen-bonding units have been discussed, offering chemical design tools to achieve supramolecular structures where excitons can be transported over a large amount of $\pi$-conjugated cores. Furthermore, the versatility of this approach, which includes multiple dyes and H-bonding functionalities, can help in the continuous search for novel materials in light-harvesting systems, electronics, and photonics.

\section{Funding Information}

LabEx Emerging Investigators Grant 2018 from the Fondation pour la Recherche en Chimie.

\section{Acknowledgments}

The authors thank the Foundation for Frontier Research in Chemistry (FRC) LabEx Emerging Investigators Grant 2018 for PhD funding and CNRS for financial support. We kindly thank Dr. Philippe Mésini for fruitful discussion.

\section{References}

(1) Lehn, J-M. Angew. Chem. Int. Ed. 1988, 27, 89.

(2) Lehn, J-M. Eur. Rev. 2009, 17, 263.

(3) Lehn, J-M. Angew. Chem. Int. Ed. 1990, 29, 1304.

(4) Fyfe, M. C. T.; Stoddart, J. F. Acc. Chem. Res. 1997, 30, 393.

(5) Stupp, S. I.; Palmer, L. C. Chem. Mater. 2014, 26, 507.

(6) Wong, K-T.; Bassani, D. M. NPG Asia Mater. 2014, 6, e116.

(7) González-Rodríguez, D.; Schenning, A. P. H. J. Chem. Mater. 2011, $23,310$.

(8) Steiner, T. Angew. Chem. Int. Ed. 2002, 41, 49.

(9) Prins, L. J.; Reinhoudt, D. N.; Timmerman, P. Angew. Chem. Int. Ed. 2001, 40, 2382.

(10) Gospodinova, N.; Tomšík, E. Prog. Polym. Sci. 2015, 43, 33.

(11) Schenning, A. P. H. J., V Herrikhuyzen J, Jonkheijm, P.; Chen, Z.; Würthner, F.; Meijer, E. W. J. Am. Chem. Soc. 2002, 124, 10252.

(12) Schoonbeek, F. S.; van Esch, J. H.; Wegewijs, B.; Rep, D. B. A.; Haas, M. d. e.; Klapwijk, T. M.; Kellogg, R. M.; Feringa, B. L. Angew. Chem. Int. Ed. 1999, 38, 1393.

(13) Würthner, F.; Thalacker, C.; Sautter, A. Adv. Mater. 1999, 11, 754.

(14) Bohanon, T. M.; Denzinger, S.; Fink, R.; Paulus, W.; Ringsdorf, H.; Weck, M. Angew. Chem. Int. Ed. 1995, 34, 58.

(15) Jelley, E. E. Nature 1937, 139, 631.

(16) Scheibe, G. Angew. Chem. 1937, 50, 212.

(17) Bücher, H.; Kuhn, H. Chem. Phys. Lett. 1970, 6, 183.

(18) Knoester, J.; Agranovich, V. M. Frenkel and charge-transfer excitons in organic solids. In: Thin Films and Nanostructures. Academic Press: Amsterdam, 2003, 1-96 (Internet: http://www. sciencedirect.com/science/article/pii/S1079405003310014).

(19) Hestand, N. J. Spano, F. C. Chem. Rev. 2018, 118, 7069.

(20) Shirakawa, M.; Kawano, S.; Fujita, N.; Sada, K.; Shinkai, S. J. Org. Chem. 2003, 68, 5037.

(21) Tani, T. Imaging Sci. J. 2007, 55, 65.

(22) Kawaguchi, T.; Iwata, K. Thin Solid Films 1990, 191, 173.

(23) Paternò, G. M.; Moretti, L.; Barker, A. J.; D’Andrea, C.; Luzio, A.; Barbero, N.; Galliano, S.; Barolo, C.; Lanzani, G.; Scotognella, F. J. Mater. Chem. C 2017, 5, 7732.

(24) Kaiser, T. E.; Stepanenko, V.; Würthner, F. J. Am. Chem. Soc. 2009, 131,6719

(25) Würthner, F.; Thalacker, C.; Diele, S.; Tschierske, C. Chem. Eur. J. 2001, 7, 2245.

(26) Crusats, J.; El-Hachemi, Z.; Escudero, C.; Ribó, J. M. J. Porphyrins Phthalocyanines 2009, 13, 461.

(27) Song, Q.; Jiao, Y.; Wang, Z.; Zhang, X. Small 2016, 12, 24.

(28) Zhou, Y.; Guzman, C.X.; Helguero-Kelley, L. C.; Liu, C.; Peurifoy, S. R.; Captain, B.; Braunschweig, A. B. J. Phys. Org. Chem. 2016, 29, 689.

(29) Ruiz-Carretero, A.; Rovelo, NRÁ.; Militzer, S.; Mésini, P. J.J. Mater. Chem. A 2019, 7, 23451.

(30) Löhner, A.; Kunsel, T.; Röhr, M. I. S.; Jansen, T. L. C.; Sengupta, S.; Würthner, F.; Knoester, J.; Köhler, J. J. Phys. Chem. Lett. 2019, 10, 2715.

(31) Brixner, T.; Hildner, R.; Köhler, J.; Lambert, C.; Würthner, F. Adv. Energy Mater. 2017, 7, 1700236.

(32) Egawa, Y.; Hayashida, R.; Anzai, J. Langmuir 2007, 23, 13146. 
(33) Fidder, H.; Terpstra, J.; Wiersma, D. A.J. Chem. Phys. 1991, 94, 6895.

(34) Ghosh, P. N. Solid State Commun. 1976, 19, 639.

(35) Scheblykin, I. G.; Bataiev, M. M.; Van der Auweraer, M.; Vitukhnovsky, A. G. Chem. Phys. Lett. 2000, 316, 37.

(36) Scheblykin, I. G.; Sliusarenko, O. Y.; Lepnev, L. S.; Vitukhnovsky, A. G.; Van der Auweraer, M. J. Phys. Chem. B 2001, 105, 4636.

(37) Dahlbom, M.; Pullerits, T.; Mukamel, S.; Sundström, V. J. Phys. Chem. B 2001, 105, 5515.

(38) Kühn, O.; Sundström, V.; Pullerits, T. Chem. Phys. 2002, 275, 15.

(39) Lin, H.; Camacho, R.; Tian, Y.; Kaiser, T. E.; Würthner, F.; Scheblykin, I. G. Nano Lett. 2010, 10, 620.

(40) Würthner, F.; Kaiser, T. E.; Saha-Möller, C. R. Angew. Chem. Int. Ed. 2011, 50, 3376.

(41) Spano, F. C. Acc. Chem. Res. 2010, 43, 429.

(42) Más-Montoya, M.; Janssen, R. A. J. Adv. Funct. Mater. 2017, 27, 1605779.

(43) Zhong, C.; Bialas, D.; Spano, F. C. J. Phys. Chem. C 2020, 124, 2146.

(44) Johansson, A.; Kollman, P.; Rothenberg, S.; McKelvey, J. J. Am. Chem. Soc. 1974, 96, 3794.

(45) Tsai, W-W.; Tevis, I. D.; Tayi, A. S.; Cui, H.; Stupp, S. I. J. Phys. Chem. B 2010, 114, 14778 .

(46) Tevis, I. D.; Tsai, W-W.; Palmer, L. C.; Aytun, T.; Stupp, S. I. ACS Nano 2012, 6, 2032.

(47) Ghosh, S.; Cherumukkil, S.; Suresh, C. H.; Ajayaghosh, A. Adv. Mater. 2017, 29, 1703783.

(48) Ghosh, S.; Das, S.; Saeki, A.; Praveen, V. K.; Seki, S.; Ajayaghosh, A. ChemNanoMat 2018, 4, 831.

(49) Kaneko, R.; Chowdhury, T. H.; Sugawa, K.; Lee, J-J.; Otsuki, J.; Islam, A. Sol. Energy 2019, 194, 248.

(50) Würthner, F. Chem. Commun. 2004, 14, 1564.

(51) Ghosh, S.; Li, X-Q.; Stepanenko, V.; Würthner, F. Chem. Eur. J. 2008, 14, 11343.

(52) Wagner, W.; Wehner, M.; Stepanenko, V.; Ogi, S.; Würthner, F. Angew. Chem. Int. Ed. 2017, 56, 16008.

(53) Ogi, S.; Stepanenko, V.; Sugiyasu, K.; Takeuchi, M.; Würthner, F.J. Am. Chem. Soc. 2015, 137, 3300.

(54) Ogi, S.; Stepanenko, V.; Thein, J.; Würthner, F. J. Am. Chem. Soc. 2016, 138, 670.

(55) Marciniak, H.; Li, X-Q.; Würthner, F.; Lochbrunner, S. J. Phys. Chem. A 2011, 115, 648.

(56) Wolter, S.; Aizezers, J.; Fennel, F.; Seidel, M.; Würthner, F.; Kühn, O.; Lochbrunner, S. New J. Phys. 2012, 14, 105027.

(57) Wolter, S.; Westphal, K. M.; Hempel, M.; Würthner, F.; Kühn, O.; Lochbrunner, S. J. Phys. B: At. Mol. Opt. Phys. 2017, 50, 184005.

(58) Fennel, F.; Wolter, S.; Xie, Z.; Plötz, P-A.; Kühn, O.; Würthner, F.; Lochbrunner, S. J. Am. Chem. Soc. 2013, 135, 18722.

(59) Ogi, S.; Fukaya, N., Arifin, Skjelstad, B. B.; Hijikata, Y.; Yamaguchi, S. Chem. Eur. J. 2019, 25, 7303.

(60) Zou, Q.; Liu, K.; Abbas, M.; Yan, X. Adv. Mater. 2016, 28, 1031.

(61) Smith, K. H.; Tejeda-Montes, E.; Poch, M.; Mata, A. Chem Soc Rev 2011, 40, 4563.

(62) Sun, Y.; Jiang, L.; Schuermann, K. C.; Adriaens, W.; Zhang, L.; Boey, F. Y. C.; De Cola, L.; Brunsveld, L.; Chen, X. Chem. Eur. J. 2011, 17, 4746.

(63) Kumar, R. J.; MacDonald, J. M.; Singh, T. B.; Waddington, L. J.; Holmes, A. B. J. Am. Chem. Soc. 2011, 133, 8564.

(64) Jintoku, H.; Sagawa, T.; Miyamoto, K.; Takafuji, M.; Ihara, H. Chem. Commun. 2010, 46, 7208.

(65) Koti, A. S. R.; Periasamy, N. Chem. Mater. 2003, 15, 369.
(66) Kim, J. H.; Lee, M.; Lee, J. S.; Park, C. B. Angew. Chem. Int. Ed. 2012 , $51,517$.

(67) Xue, B.; Li, Y.; Yang, F.; Zhang, C.; Qin, M.; Cao, Y.; Wang, W. Nanoscale 2014, 6, 7832.

(68) Kim, J. H.; Nam, D. H.; Lee, Y. W.; Nam, Y. S.; Park, C. B. Small 2014, 10, 1272.

(69) Smith, A. M.; Williams, R. J.; Tang, C.; Coppo, P.; Collins, R. F.; Turner, M. L.; Saiani, A.; Ulijn, R. V. Adv. Mater. 2008, 20, 37.

(70) Shimizu, L. S.; Smith, M. D.; Hughes, A. D.; Shimizu, K. D. Chem. Commun. 2001, 1592.

(71) Shimizu, L. S.; Salpage, S. R.; Korous, A. A. Acc. Chem. Res. 2014, 47, 2116.

(72) Simic, V.; Bouteiller, L.; Jalabert, M.J. Am. Chem. Soc. 2003, 125, 13148.

(73) Isare, B.; Pensec, S.; Raynal, M.; Bouteiller, L. C. R. Chim. 2016, 19, 148.

(74) Das, A.; Ghosh, S. Chem. Eur. J. 2010, 16, 13622.

(75) Liu, Y.; Liu, L.; Zhu, E.; Yue, M.; Gao, C.; Wu, X.; Che, G.; Liu, H. ChemPlusChem 2018, 83, 1109.

(76) Shoji, S.; Ogawa, T.; Hashishin, T.; Tamiaki, H. ChemPhysChem 2018, 19, 913.

(77) Shoji, S.; Ogawa, T.; Matsubara, S.; Tamiaki, H. Sci. Rep. 2019, 9, 14006.

(78) Kar, H.; Gehrig, D. W.; Allampally, N. K.; Fernández, G.; Laquai, F.; Ghosh, S. Chem. Sci. 2016, 7, 1115.

(79) Yagai, S.; Kitamoto, Y.; Datta, S.; Adhikari, B. Acc. Chem. Res. 2019, $52,1325$.

(80) Yagai, S.; Goto, Y.; Lin, X.; Karatsu, T.; Kitamura, A.; Kuzuhara, D.; Yamada, H.; Kikkawa, Y.; Saeki, A.; Seki, S. Angew. Chem. Int. Ed. 2012, 51, 6643.

(81) Adhikari, B.; Yamada, Y.; Yamauchi, M.; Wakita, K.; Lin, X.; Aratsu, K.; Ohba, T.; Karatsu, T.; Hollamby, M. J.; Shimizu, N.; Takagi, H.; Haruki, R.; Adachi, S.; Yagai, S. Nat. Commun. 2017, 8, 15254.

(82) Iwaura, R.; Ohnishi-Kameyama, M.; Iizawa, T. Chem. Eur. J. 2009, $15,3729$.

(83) Mandal, S.; Zhou, X.; Lin, S.; Yan, H.; Woodbury, N. Bioconjugate Chem. 2019, 30, 1870.

(84) Banal, J. L.; Kondo, T.; Veneziano, R.; Bathe, M.; Schlau-Cohen, G. S. J. Phys. Chem. Lett. 2017, 8, 5827.

(85) Boulais, É.; Sawaya, N. P. D.; Veneziano, R.; Andreoni, A.; Banal, J. L.; Kondo, T.; Mandal, S.; Lin, S.; Schlau-Cohen, G. S.; Woodbury, N. W.; Yan, H.; Aspuru-Guzik, A.; Bathe, M. Nat. Mater. 2018, 17, 159.

(86) Markova, L. I.; Malinovskii, V. L.; Patsenker, L. D.; Häner, R. v. S. Chem. Commun. 2013, 49, 5298.

(87) Zhou, Y.; Guzman, C. X.; Helguero-Kelley, L. C.; Liu, C.; Peurifoy, S. R.; Captain, B.; Braunschweig, A. B.J. Phys. Org. Chem. 2016, 29, 689.

(88) Levine, A. M.; Schierl, C.; Basel, B. S.; Ahmed, M.; Camargo, B. A.; Guldi, D. M.; Braunschweig, A. B. J. Phys. Chem. C 2019, 123, 1587.

(89) Villari, V.; Mineo, P.; Scamporrino, E.; Micali, N. RSC Adv. 2012, 2, 12989.

(90) Militzer, S.; Tran, T. M. P.; Mésini, P. J.; Ruiz-Carretero, A. ChemNanoMat 2018, 4, 790.

(91) Herbst, S.; Soberats, B.; Leowanawat, P.; Lehmann, M.; Würthner, F. Angew. Chem. Int. Ed. 2017, 56, 2162.

(92) Herbst, S.; Soberats, B.; Leowanawat, P.; Stolte, M.; Lehmann, M.; Würthner, F. Nat. Commun. 2018, 9, 2646.

(93) Hecht, M.; Schlossarek, T.; Stolte, M.; Lehmann, M.; Würthner, F. Angew. Chem. Int. Ed. 2019, 58, 12979. 